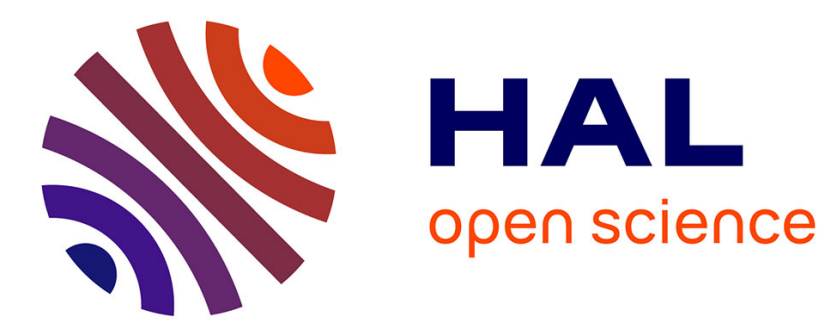

\title{
Mieux comprendre les comportements alimentaires grâce au concept de perception du risque nutritionnel
}

Marie-Eve Laporte, Géraldine Michel, Sophie Rieunier

\section{To cite this version:}

Marie-Eve Laporte, Géraldine Michel, Sophie Rieunier. Mieux comprendre les comportements alimentaires grâce au concept de perception du risque nutritionnel. Recherche et Applications en Marketing (French Edition), 2015, 30 (1), pp.81-117. 10.1177/0767370114550443 . hal-02054434

\section{HAL Id: hal-02054434 \\ https://hal.science/hal-02054434}

Submitted on 1 Mar 2019

HAL is a multi-disciplinary open access archive for the deposit and dissemination of scientific research documents, whether they are published or not. The documents may come from teaching and research institutions in France or abroad, or from public or private research centers.
L'archive ouverte pluridisciplinaire HAL, est destinée au dépôt et à la diffusion de documents scientifiques de niveau recherche, publiés ou non, émanant des établissements d'enseignement et de recherche français ou étrangers, des laboratoires publics ou privés. 
Article RAM janvier 2015 (volume $30 n^{\circ} 1$ )

Mieux comprendre les comportements alimentaires grâce au concept de perception du risque nutritionnel

Marie-Eve Laporte

Laboratoire GREGOR, IAE Paris I Panthéon Sorbonne

Géraldine Michel

Laboratoire GREGOR, IAE Paris I Panthéon Sorbonne

Sophie Rieunier

Laboratoire GREGOR, IAE Paris I Panthéon Sorbonne

Les auteurs remercient le rédacteur en chef Joël Brée et les trois lecteurs anonymes pour leurs commentaires constructifs qui ont profondément amélioré cet article. 


\section{Mieux comprendre les comportements alimentaires grâce au concept de perception du risque nutritionnel}

RESUME : Le comportement nutritionnel des populations est une priorité pour les pouvoirs publics. En effet, les maladies associées sont la première cause de mortalité dans le monde. Dans un souci de santé publique, il est essentiel de mieux comprendre la Perception du Risque Nutritionnel par le consommateur (PRN), car elle influence l'intention comportementale d'après le modèle de la motivation à se protéger (Rogers, 1975 ; Maddux et Rogers, 1983). A travers une synthèse de la littérature et une étude qualitative, cet article clarifie le concept de PRN, explore de potentiels facteurs explicatifs ou modérateurs, et identifie des pistes de recherche. Il montre que la PRN ne tient pas seulement aux caractéristiques du produit (rôle de la naturalité perçue), mais aussi au contexte de consommation (rôle de la commensalité) et d'achat (rôle de l'hyperchoix).

Mots-clés : Alimentation, allégations nutritionnelles, commensalité, comportement du consommateur, hyperchoix, hyperchoix, naturalité, risque nutritionnel

\section{Toward a better understanding of eating-behaviour through the concept of Perception of Nutritional Risk}

ABSTRACT: The general public's nutritional behaviour is a priority of public-health authorities. Associated diseases are the prime cause of mortality worldwide. For reasons of public health, a better understanding of the consumer Perception of Nutritional Risk (PNR) is essential, since this, according to the protection motivation theory, influences behavioural intention (Maddux and Rogers, 1983; Rogers, 1975). Following a review of the literature and qualitative research, the present article endeavours to clarify the concept of PNR, explore potential explanatory or moderating factors, and identify leads for future research. We show that PNR depends not solely on the product characteristics (role of perceived naturalness), but also the consumption situation (role of commensality) and purchasing conditions (role of hyperchoice).

Keys words: Food, nutrition claims, commensality, consumer behaviour, hyperchoice, naturalness, nutritional risk 


\section{Mieux comprendre les comportements alimentaires grâce au concept de perception du risque nutritionnel}

\section{INTRODUCTION}

La nutrition compte parmi les priorités des pouvoirs publics en raison du coût humain et économique croissant des «maladies nutritionnelles » chroniques, ainsi qualifiées par la FAO - l'organisation des nations unies pour l'alimentation et l'agriculture -, comme les maladies cardio-vasculaires, l'obésité, le diabète, et certains cancers (Latham, 2001). Elles sont à l'origine d'environ 60\% des décès dans le monde (OMS et FAO, 2003). Qualifiées à tort de «maladies de riches », elles progressent à un rythme alarmant dans les pays en voie de développement, et touchent principalement les populations pauvres dans les pays industrialisés (Latham, 2001; OMS et FAO, 2003). Or, l’industrie agroalimentaire est directement accusée de contribuer au développement de ces maladies et de la plus visible d’entre elles, l’obésité (Brée, 2010 ; Moore et Rideout, 2007). En réaction à ces critiques, de nombreuses marques ont développé l'usage d'allégations nutritionnelles ${ }^{1}$ à la suite du yaourt Bio (Activia) de Danone, pionnier en 1987, même si le règlement européen n¹924/2006 en a restreint les conditions d'utilisation depuis. On aurait pu s'attendre à une amélioration des comportements alimentaires de la part d’individus désormais mieux informés. Pourtant, ce n’est pas le cas. Selon certaines recherches centrées sur l’obésité, les allégations nutritionnelles montrent des effets pervers néfastes à la santé des individus, par exemple une augmentation importante des quantités consommées quand le produit est affiché comme allégé (Nestle, 2007 ; Wansink et Chandon, 2006).

Au cœur de ce paradoxe, un concept paraît central, la Perception du Risque Nutritionnel par le consommateur (PRN). Le risque nutritionnel, composante long terme du risque alimentaire (Aurier et Sirieix, 2009), se manifeste par les effets secondaires des aliments ingérés (Ferreira, 2006) - par exemple des carences ou une prise ou perte de poids

1 Le règlement européen $n^{\circ} 1924 / 2006$ définit l'allégation comme «tout message ou toute représentation, non obligatoire en vertu de la législation communautaire ou nationale, y compris une représentation sous la forme d'images, d'éléments graphiques ou de symboles, qu'elle qu'en soit la forme, qui affirme, suggère ou implique qu'une denrée alimentaire possède des caractéristiques particulières » (art. 2.2.1) ; pour une allégation nutritionnelle, ce sont les " propriétés nutritionnelles bénéfiques particulières de par l'énergie (valeur calorique) qu'elle fournit, fournit à un degré moindre ou plus élevé ou ne fournit pas, les nutriments ou autres substances qu'elle contient, contient en proportion moindre ou plus élevée ou ne contient pas » (art. 2.2.4) ; pour une allégation de santé, c'est l' «existence d'une relation entre, d'une part, une catégorie de denrées alimentaires, une denrée alimentaire ou l'un de ses composants, et, d'autre part, la santé » (art. 2.2.5). 
inappropriée -, sur la santé définie comme « état de complet bien-être physique, mental et social » (OMS, 1946). Pour éclairer les comportements des individus confrontés à un risque alimentaire, des travaux montrent l'intérêt de distinguer la «perception du risque » de l' «attitude face au risque » (Moon et Balasubramanian, 2003 ; Pennings, Wansink et Meulenberg, 2002). La perception du risque est l'interprétation par les consommateurs de leur exposition à ce risque (Pennings, Wansink et Meulenberg, 2002). L'attitude face au risque est leur prédisposition générale à prendre ce type de risque (Pennings, Wansink et Meulenberg, 2002). Certaines recherches étudient certes l'attitude face au risque pour expliquer la prise de risque nutritionnel, distinguant par exemple les consommateurs en recherche de bien-être orientés promotion - de ceux cherchant à éviter les maladies - orientés prévention - (Gomez, 2009b ; Gurviez, 2010 ; Werle et al., 2012). Mais ces travaux n’intègrent pas la PRN.

Pourtant, modifier les comportements nécessite d'influencer la perception du risque. En effet, dans le modèle de la motivation à se protéger de Rogers, la probabilité d'occurrence (vulnérabilité) et la sévérité de la menace sont des préalables à l'intention de se protéger (Maddux et Rogers, 1983 ; Rogers, 1975). Or, ce sont justement les deux dimensions du risque perçu (Bauer, 1960), comme illustré dans la Figure 1.

[Figure 1]

La PRN apparaît donc comme un antécédent de l'intention comportementale, « indicateur de la volonté d'essayer, de consentir à un effort pour se comporter d'une façon donnée » (Ajzen, 1991). De cette intention comportementale découle le comportement, non seulement dans le modèle de Rogers, mais aussi dans la théorie de l'action raisonnée (Fishbein et Ajzen, 1975 ; Ajzen et Fishbein, 1980) ou dans la théorie du comportement planifié qui en est issue (Ajzen, 1991). Dès lors, dans l'objectif d'améliorer le comportement alimentaire des populations et pour notamment limiter les carences et ajuster les prises caloriques, il serait utile de comprendre la PRN et ses mécanismes: comment les consommateurs évaluent-ils leur exposition au risque nutritionnel des produits ? Quels facteurs influencent leur PRN ?

Pour répondre à ces questions, cet article synthétise d'abord les travaux de recherche liés à la Perception du Risque Nutritionnel. Il les complète par une étude qualitative menée auprès de dix-huit consommateurs. Il met ensuite les résultats en perspective, propose des pistes de recherche et présente ses limites. Sur un plan académique, il clarifie la PRN et suggère un lien avec trois concepts qui touchent le marketing, chacun dans une catégorie différente : parmi les variables relatives au produit, la naturalité perçue; parmi celles tenant à la situation de consommation, la commensalité ; et parmi celles liées à l'environnement d'achat, 
l'hyperchoix. Enfin, dans une perspective managériale sociale, cet article alerte les pouvoirs publics sur les mécanismes sous-jacents de la PRN, dans une optique d'améliorer le comportement nutritionnel des populations.

\section{LA PERCEPTION DU RISQUE NUTRITIONNEL PAR LE CONSOMMATEUR}

La Perception du Risque Nutritionnel est un concept récent encore peu traité dans la littérature marketing. Pour mieux l'appréhender, il est apparu nécessaire d'explorer des champs de recherche connexes, comme la sociologie et la psychologie de l'alimentation, l'économie, la psychologie et la sociologie du risque, le marketing agro-alimentaire, ou la nutrition.

\section{L'alimentation, une consommation anxiogène}

L'aliment n'est pas un bien de grande consommation comme les autres (Poulain, 2002). A côté de son caractère hédonique et social, l'alimentation comporte un risque biologique, inhérent à l'ingestion vitale et multi-quotidienne de nourriture (Kapferer, 1998 ; Rozin, 2005b). De plus, elle repose sur le «principe d'incorporation » (Fischler, 1990), qui participe à la construction de l'identité dès la petite enfance (Gurviez, 2001), et transforme l'individu de l'intérieur. En effet, l'être humain assimile certes les nutriments présents dans l'aliment, mais aussi les représentations qu’il s’en fait (Fischler, 1990), par « pensée magique » (Frazer, 1911). Chaque société construit donc ses propres références de ce qui est comestible et de ce qui est impropre à la consommation (Merdji, 2006). Enfin, l'être humain est confronté à une contradiction insurmontable, le « paradoxe de l'homnivore » (Fischler, 1990 ; Fischler, 2001). L’homme doit être néophobe pour se protéger d'aliments qui pourraient le rendre malade ou même le tuer. Mais il doit aussi être néophile pour assurer la diversité nutritionnelle nécessaire à son maintien en bonne santé. Toutes ces caractéristiques propres à la consommation alimentaire mettent en évidence son caractère anxiogène, même si le plaisir et l'échange social restent encore essentiels dans les représentations de l'alimentation en France selon la « loi des $2 \mathrm{~S}$ : Sécurité-Santé dans les pays anglo-saxons, Saveur-Sociabilité ailleurs » (Ferrières, 2006). L’alimentation renvoie donc au concept de risque.

\section{Le risque nutritionnel}

Le concept de risque est complexe, d'abord par son double caractère objectif et subjectif (Douglas et Wildavsky, 1983 ; Kahneman et Tversky, 1979 ; Rosa, 2003). C’est « en partie une menace ou un préjudice objectif envers les gens et en partie le produit d'une expérience 
culturelle et sociale » (Kasperson in Pidgeon, Kasperson et Slovic, 2003). Une autre difficulté tient au caractère multidimensionnel du risque perçu (Mitchell, 1999 ; Volle, 1995), à la fois physique, psychologique, social, financier et de performance (Cox, 1967 ; Kaplan, Szybillo et Jacoby, 1974), de perte de temps (Cox, 1967 ; Roselius, 1971) et éthique (Aurier et Sirieix, 2009 ; Douglas et Wildavsky, 1983 ; Kermisch, 2011 ; Kytle et Ruggie, 2005 ; Méric, Pesqueux et Solé, 2009). Compte-tenu du caractère vital de la nourriture, c'est la dimension physique qui prévaut dans le risque alimentaire (Aurier et Sirieix, 2009 ; Bergadaà et Urien, 2006 ; Gallen, 2001). Lui-même se décompose en risque sanitaire d'empoisonnement à court terme - par contamination de l'aliment -, et risque nutritionnel d'effets secondaires à long terme (Aurier et Sirieix, 2009 ; Ferreira, 2006), comme l'illustre le tableau 1. La PRN exprime quant à elle «une incertitude liée aux conséquences négatives d'un choix » alimentaire (Aurier et Sirieix, 2009), à long terme sur sa santé physique, mentale et sociale.

[Tableau 1]

Le risque nutritionnel est causé par des déséquilibres, carences ou excès, comme l'exposition répétée à des ingrédients à la longue nocifs, seuls ou associés à d'autres par « effet cocktail » (Chevallier, 2009). A l'extrême, il peut provoquer des maladies nutritionnelles qui affectent la longévité ou la qualité de vie de l'individu. Cependant, ses conséquences ne sont pas seulement physiques. Elles touchent aussi le bien-être psychologique et l'identité sociale de l'individu, et revêtent ainsi une dimension psychosociale (Bergadaà et Urien, 2006). Alors que la collectivité prend en charge le risque sanitaire, elle laisse le risque nutritionnel à l'appréciation de l'individu, jugé responsable de ses choix nutritionnels (Fischler, 1990 ; Fischler et Masson, 2008 ; Lipovetsky, 2006).

Toutefois, les individus ne manifestent pas tous la même attitude face au risque nutritionnel. Par exemple, les femmes - a fortiori avec enfants, et les personnes âgées ou confrontées à la maladie se soucient plus de nutrition que les autres (Fischler et al., 2010 ; Grunert et Wills, 2007 ; Zingg et al., 2013), car les femmes ont la responsabilité d’autrui, et les personnes âgées une perception du temps altérée (Bech-Larsen et Scholderer, 2007). Au contraire, les adolescents montrent une tendance générale à rechercher le risque (Camous, 2011), car il peut constituer un rite de passage vers l'âge adulte (Lardellier, 2011). Les adolescents sont peu réceptifs à la nutrition dont ils jugent les conséquences trop éloignées (Werle et al., 2012). Il s’avère donc nécessaire de prendre en compte à la fois l'attitude de l'individu face au risque nutritionnel et la PRN pour expliquer la prise de risque nutritionnel. En effet, d'après le modèle de la motivation à se protéger présenté en Figure 1, le consommateur doit percevoir 
un risque nutritionnel pour adopter un comportement préventif (Rogers, 1975 ; Maddux et Rogers, 1983). Mais cette condition nécessaire n’est pas suffisante : l’individu peut malgré la PRN accepter de prendre le risque, en fonction de sa propre attitude face au risque (Pennings, Wansink et Meulenberg, 2002).

\section{L’influence ambiguë des allégations nutritionnelles sur la PRN}

Parce qu'il est difficile d'évaluer les caractéristiques nutritionnelles des aliments, les consommateurs ont tendance à utiliser des heuristiques de choix (Gomez, 2009a ; 2009b ; Larceneux, 2003b). Ces raccourcis décisionnels permettent de simplifier et d'accélérer le processus de décision (Tversky et Kahneman, 1992). Pour cela, les consommateurs peuvent s’appuyer sur des indicateurs de qualité intrinsèques au produit, comme l'apparence (Brunel et Pichon, 2004 ; Sirieix, 1999), ou extrinsèques au produit, comme la marque (Kapferer, 1998 ; Michel, 2004), les labels (Larceneux, 2003b ; 2003a), et de plus en plus souvent, les allégations nutritionnelles (Gomez, 2008). Par exemple, les biscuits Prince de Lu mettent en avant les mentions «sans colorant, sans conservateur, sans matière grasse hydrogénée, riches en céréales et au lait ». Comment les allégations nutritionnelles influencent-elles le comportement alimentaire? Des recherches montrent l'existence d'effets ambigus (voir en annexe A1 la synthèse des principaux articles considérés). Il convient toutefois de distinguer les résultats selon l'origine des travaux. En effet, il existe de fortes différences culturelles dans la relation au risque nutritionnel entre Etats-Unis, Europe du Nord et Europe du Sud (Fischler et Masson, 2008 ; Fischler et al., 2010 ; Grunert et Wills, 2007 ; Rozin et al., 2006). Ainsi, l'alimentation apparaît individualisée et médicalisée aux Etats-Unis, sociale et conviviale en France par héritage culturel (Fischler et Masson, 2008).

Dans plusieurs expérimentations menées aux Etats-Unis, la présence d'allégations nutritionnelles conduit à une prise de calories accrue mais inconsciente (Chandon et Wansink, 2010). En effet, le consommateur classe les aliments de façon dichotomique en bons ou mauvais pour la santé, sans se référer aux doses (Chernev et Gal, 2010). Ainsi, une expérimentation montre qu'en présence de M\&Ms à « faible teneur en matières grasses », les consommateurs augmentent la taille des portions et la prise énergétique (Wansink et Chandon, 2006). La présence d’allégations nutritionnelles semble alors inhiber la PRN. Ce phénomène est amplifié chez les personnes en surpoids (Wansink et Chandon, 2006). Dès lors, certains chercheurs font le parallèle entre l'industrie agroalimentaire et celle du tabac (Nestle, 2007) : alors que les cigarettes light étaient censées réduire la dépendance et les risques, les fumeurs 
déculpabilisés en fument plus et aspirent plus fortement, avec un risque de développer des cancers plus bas dans les poumons (Brownell et Warner, 2009). Parallèlement, les aliments light supposés diminuer la prise énergétique peuvent entraîner une absorption de calories supérieure (Nestle, 2007 ; Wansink et Chandon, 2006), encore plus importante chez les personnes déjà en surpoids du fait de la taille accrue de leurs portions (Chandon et Wansink, 2007a).

A l'inverse, en France, les allégations nutritionnelles semblent augmenter la PRN. Ainsi, les Français attribuent à un simple yaourt nature une meilleure note santé qu'à une version allégée ou enrichie avec un ingrédient bénéfique pour la santé (Fischler et al., 2010). De même, le jambon artisanal est jugé plus sain que les formats allégé ou fortifié en oméga 3 (Merdji, 2012). La différence culturelle d'attitude envers les allégations nutritionnelles peut contribuer à expliquer cette différence entre Etats-Unis et France (Grunert et Wills, 2007). Mais dans les deux cas, les allégations nutritionnelles semblent biaiser la PRN, dans un sens ou dans l'autre.

\section{La méfiance des consommateurs face aux allégations nutritionnelles}

L’utilisation des allégations nutritionnelles comme indicateur de qualité suppose que les consommateurs aient confiance en cette «qualité communiquée » (Brunel et Pichon, 2004 ; Sirieix, 1999 ; Sirieix et Dubois, 1999). Or, des recherches soulignent le scepticisme des consommateurs face au discours santé des marques (Gurviez, 2010 ; Keller et al., 1997). En effet, la nutrition repose sur des attributs de croyance, donc non vérifiables par le consommateur (Gomez, 2009b ; Larceneux, 2003b ; Larceneux, 2003a ; Mason, 2009). Les allégations nutritionnelles sont perçues par les consommateurs comme des discours marketing (Burton et Creyer, 2004 ; Kozup, Creyer et Burton, 2003). Elles ne sont donc pas considérées comme une source d'information nutritionnelle crédible car aucune des deux conditions requises n’est remplie, ni la compétence, ni l’intégrité (Simon, Berkowitz et Moyer, 1970). Premièrement, le marketing n'est pas perçu comme un expert nutritionnel du fait de la « cacophonie alimentaire » (IREMAS, 2010). Deuxièmement, l’intégrité des marketeurs est mise en cause par un consommateur de plus en plus éduqué, conscient de la divergence d'intérêt entre sa propre recherche de qualité nutritionnelle et l'objectif de profit de l'entreprise (Singh et Sirdeshmukh, 2000). Dès lors, les valeurs nutritionnelles formatées par les pouvoirs publics sont jugées plus crédibles que les allégations, qui sont attribuées aux 
marques et considérées comme des promesses peu fiables (Burton et Creyer, 2004 ; Kozup, Creyer et Burton, 2003).

En France, cette méfiance semble encore plus forte car elle remet en cause les représentations sociales de l'alimentation et l' «identité collective » (Gurviez, 2010), reposant sur un socle historique et culturel partagé (Ferrières, 2006 ; Fischler et Masson, 2008 ; Flandrin et Montanari, 1996). Ainsi, une netnographie des commentaires négatifs sur Actimel de Danone montre la résistance de ces consommateurs face aux allégations de renforcement des défenses immunitaires $^{2}$ car elles sont incongruentes avec le modèle d'alimentation français, qu'elles mettent en danger (Gurviez, 2010). Dans une perspective d'anti-consommation, ces antiActimel dénoncent un « coup marketing » qui vise à enrichir l'entreprise aux dépens des plus vulnérables par de la manipulation affective et de la culpabilisation (Gurviez, 2010).

En conclusion de cette revue de littérature, la Figure 2 synthétise les relations supposées entre les variables rencontrées. Pour éclairer le comportement des consommateurs-mangeurs et améliorer la santé publique, il apparaît essentiel de mieux comprendre la PRN et ses mécanismes. L'étude qualitative suivante s'emploie donc à identifier d'autres potentiels facteurs susceptibles d'influencer la PRN ou la relation entre PRN et intention comportementale.

[Figure 2]

\section{ETUDE QUALITATIVE}

La revue de littérature a montré la complexité de la PRN et le rôle ambigu des allégations nutritionnelles. Mais comment les consommateurs évaluent-ils leur exposition au risque nutritionnel des produits? Quels facteurs influencent leur PRN? Pour approfondir les réponses à ces questions posées en introduction, une étude qualitative a été conduite auprès de consommateurs.

\section{Méthodologie}

Des entretiens individuels, chacun d'environ une heure trente, ont été menés auprès de dixhuit hommes et femmes d’âges et de conditions sociales variés (voir la composition de l'échantillon en annexe A2). Le nombre retenu de dix-huit répondants a découlé du principe

\footnotetext{
${ }^{2}$ A noter, les allégations d'Actimel ont dû être modifiées en 2010 suite à l'application de la législation européenne EC1924/2006.
} 
de saturation (Evrard et al., 2009). Ces entretiens se sont déroulés en immersion dans la cuisine des individus, afin de les mettre à l'aise et de pouvoir observer leur environnement. En effet, sur des sujets sensibles, l'interviewé se sent souvent plus détendu lorsqu'il est interrogé chez lui (Gavard-Perret et al., 2008). De plus, cela permet de coupler observation et rapports verbaux (Arnould et Wallendorf, 1994). Les entretiens, d'abord non directifs sur le thème des angoisses alimentaires, ont ensuite mobilisé des techniques projectives dans un souci de facilitation (Evrard et al., 2009). Un premier jeu de photographies a été présenté, illustrant neuf situations associées à l'alimentation rencontrées notamment dans la littérature sociologique (Boutaud, 2004 ; Fischler et Masson, 2008 ; Lahlou, 1995), comme le plaisir, l'angoisse ou la convivialité. L’objectif était triple : vérifier la présence de peurs alimentaires, distinguer risques sanitaire et nutritionnel, et identifier des images émotionnelles associées. Un second jeu de photographies a ensuite été ajouté au premier, montrant huit plats traditionnels, par exemple le cassoulet ou la tarte Tatin. Cela a permis de savoir si la PRN est différente selon que le répondant visualise ou non le plat, et d'explorer les représentations associées dans ces deux situations. L'entretien s'est conclu par une liste de thèmes relatifs à l'alimentation s’ils n’avaient pas été abordés (voir le guide d'entretien en annexe A3). Quelques semaines plus tard, l'analyse des tickets de caisse des courses alimentaires et des notes de restaurant accumulés dans l'intervalle a permis d'estimer les budgets alimentaires des personnes dans un souci de contrôle de la variable et de vérification du déclaratif.

Les entretiens ont été intégralement enregistrés et retranscrits, puis traités selon deux méthodes successives. Une analyse transversale par «attention flottante » a d'abord fait émerger des thématiques communes (Ayache et Dumez, 2011a ; 2011b) : en sus du rôle des allégations nutritionnelles sont apparus la naturalité perçue, la commensalité, et l’hyperchoix. Ces thèmes ont ensuite guidé l'analyse de contenu systématique réalisée à l'aide du logiciel NVivo8. Les principaux verbatim sont disponibles dans les annexes A4 à A7.

\section{Résultats}

\section{Une perception de risque plutôt nutritionnel que sanitaire}

En premier lieu, la totalité des dix-huit personnes interviewées relie alimentation et santé : «je suis intimement convaincue que tu peux améliorer ta santé par l'alimentation, et la détruire aussi » (Sylvie). Les hommes apparaissent un peu moins inquiets, car les femmes ont généralement la responsabilité de nourrir la famille: «intellectuellement, oui, mais concrètement, est-ce que je pense à ma santé quand je mange, je dois dire que non. (...) 
J'aurais tendance à me reposer sur [ma femme] pour ça, c’est elle qui fait les menus » (JeanMarc). Seules deux personnes parmi les répondants n’affichent aucune inquiétude par rapport à leur alimentation, même si elles établissent un lien théorique entre alimentation et santé. Pourtant, toutes deux sont des femmes âgées, a priori plus sensibles à la nutrition. Ce qui les différencie des autres répondants, c’est qu'elles ont connu la faim pendant la guerre. Cela leur fait relativiser leur alimentation, alors que «les gens qui n'ont pas vu la guerre, (...) comme ils n'ont pas souffert de la faim, ils sont plus regardants » (Madeleine).

Même si les entretiens ont été réalisés pendant des crises sanitaires fortement médiatisées ${ }^{3}$, les inquiétudes alimentaires exprimées portent presque toutes sur le risque nutritionnel. En effet, pour les répondants, la sécurité alimentaire est aujourd'hui bien maîtrisée en France. De plus, certaines mesures d'hygiène préviennent le risque sanitaire: «Dans cette affaire du concombre, si tu as une bonne hygiène, tu laves, tu enlèves la peau et tout, tu ne risques rien » (Marie-Claude). «Les biftecks hachés, il faut bien les cuire » (Madeleine). En revanche, les répondants sont plus dépourvus face au risque nutritionnel : «dans la nourriture sophistiquée, il y a des inconvénients qui sont beaucoup plus tendancieux parce que tu as les ferments qui sont installés, et tu sais pas comment ils réagiront dans plusieurs années » (Claude). «On sait plus maintenant ce qu'est une nourriture saine parce qu'on ne sait plus quoi manger! Tout est devenu dangereux. (...) Je m'inquiète des effets à long terme, des nourritures cancéreuses, qui sont cancéreuses à long terme " (Chantal). Toutefois, un tiers des répondants doute qu'une mauvaise nutrition puisse aller jusqu'à déclencher de graves maladies: «Incontestablement il y a un lien entre alimentation et santé, (...) mais moi j’associe quand même pas si facilement que ça maladie grave et alimentation " (Evelyne). "Ça donne un terrain plus propice, ou moins propice. Après, être tombé malade de mal manger, je pense que faut vraiment être dans l'excès » (Adeline). Les effets nutritionnels à long terme sur la santé prise au sens large sont donc source d'inquiétude. Notons cependant que la frontière entre risque sanitaire et risque nutritionnel n'est pas toujours très claire pour les répondants (voir en annexe A3 la grille des risques alimentaires mentionnés). Ainsi, la présence légalement autorisée d’OGM ou de pesticides sous les seuils réglementaires européens (Maximum Residue Levels) est qualifiée de risque sanitaire par près de la moitié des répondants, malgré l’absence de risque avéré à court terme.

\footnotetext{
${ }^{3}$ De mai à juillet 2012 : e-coli en Allemagne due à des graines germées bio, initialement attribuée au concombre espagnol (29 occurrences parmi les répondants) ; puis e-coli en France suite à des steaks hachés avariés.
} 
Quels sont les mécanismes de la PRN ? D’après cette recherche qualitative, elle n’est pas influencée seulement par les caractéristiques du produit, mais aussi par la situation de consommation et l'environnement d'achat.

Parmi les variables liées au produit, la naturalité perçue

La naturalité perçue, ou caractère naturel attribué par les individus aux aliments, est largement évoquée par les répondants, comme le montrent le tableau 2 et les verbatim en annexe A4.

[Tableau 2]

Ainsi, les aliments vus comme naturels sont considérés plus sains : «si je pars de produits simples, je me dis que je mange plus sainement que si je prends des produits manufacturés » (Sylvie). La naturalité perçue semble diminuer la PRN : «Un gâteau fait-maison, oui. (...) C'est pas un gâteau industriel, (...) c'est mieux quoi. Même pour la santé. (...) Tout ce qui est industriel, c'est jamais bon pour la santé, hein » (Lucie). Cela pose la question des allégations supposant une transformation de l'aliment, de type « enrichi en » ou « allégé en ». Toutefois, les consommateurs interrogés ne perçoivent pas de la même façon l'ajout et le retrait. Dans leurs propos, le terme « enrichi en » est plus mal vu que « décaféiné » ou " allégé en » car il pose la question de ce qui est mis : «c'est bien beau de marquer riche en oméga trois, mais il faut voir d'où ils viennent » (Claude). Il suppose de plus une intervention technique : «Si y a un jus de fruit enrichi en fer, je prends pas. Je préfère un jus de fruit normal, sans rien d'ajouté » (Marie).

A quoi les consommateurs reconnaissent-il un produit «naturel » ? Les répondants mettent en avant les ingrédients et le procédé de transformation, comme l'indique le tableau 2. «Pour que ce soit sain, il faut le moins d'ingrédients possible » (Thierry). Faire la cuisine permet de réduire la perception de risque nutritionnel, car on contrôle non seulement les ingrédients, mais aussi la fabrication. D’autre part, si l’aliment est "calibré », «standardisé », cela l'éloigne de la nature et génère des doutes sur son mode de production. Ce n’est «pas forcément un produit fait pour être bon, mais un produit fait pour être beau. A la limite, c'est suspect » (Claude). Enfin, les répondants expriment une préférence pour les produits de saison et pour les achats chez les petits commerçants plutôt que dans la grande distribution, associée à la production industrialisée, et qui «fait peur » (Audrey). Ce discours est à nuancer car l'analyse des tickets de caisse montre que l'essentiel de leurs courses est de fait réalisé en supermarché, au moins pour l'épicerie. Mais effectivement, la viande et les fruits et légumes sont souvent achetés chez de petits commerçants. De plus, en observant le garde-manger des 
répondants, on a pu constater une large présence de produits transformés, dont certains comportaient une longue liste d'ingrédients, contredisant ainsi en partie leurs propos : «Là normalement, y a rien d'ajouté, si j'ai bien choisi... Où est-ce qu'il y a les ingrédients, faut les chercher les ingrédients : chou-fleur, carottes, pois croquants, courgettes, eau, ah, graisse végétale, beurre, bouillon de légume, ah oui non non, amidon de riz, sel, épices. (...) C'est quand même incroyable : je pense que j'ai pas dû regarder là. (...) Ca ça m'agace, hein » (Marie). La recherche de naturalité ne résiste donc pas toujours à l'épreuve des faits. Soumis à des contraintes de temps ou d'argent, les consommateurs font preuve de pragmatisme. Mais déroger à leur idéal semble une source additionnelle de stress et de PRN.

Parmi les variables liées à la situation de consommation, la commensalité Au-delà des aliments, leur situation de consommation joue un rôle important dans la perception des répondants, en témoignent le tableau 3 et les verbatim en annexe A5.

[Tableau 3]

Ainsi, les répondants associent une alimentation grasse ou sucrée à des situations négatives, comme la maladie ou l'angoisse. Pourtant, face à des images de plats gras ou sucrés, les évocations redeviennent positives : la joie de vivre, la convivialité... « Manger, partager, (...) ça va ensemble. Si tu manges tout seul, c'est pas drôle. (...) [En France quand on parle de manger], on parle plus de partager. Et du coup c'est plus une source de stress. Quand tu partages, tu peux te dire que tu manges beaucoup, ça, ça te stresse pas. Alors que je pense si tu es tout seul et que tu manges beaucoup, là ça te stresse » (Hervé).

En réalité, plus que ce qui est partagé - le contenu des plats -, c’est l'opportunité de partager qui semble prévaloir. Treize des dix-huit répondants ont spontanément évoqué la dimension sociale de la nourriture dans la partie non directive des entretiens : "C'est pas tant lié à ce qu'on mange qu'à la, la circonstance quoi. Par exemple, quand j'ai des copains qui viennent à la maison, finalement j'ai tendance à leur faire à manger quelque chose qu'est pas très différent de ce que je mange moi, mais l'ambiance est très différente parce que c'est une convivialité autour » (Thierry). D’ailleurs, même si la qualité des aliments n’est pas au rendez-vous, le rôle bénéfique du partage est souligné : «la cantine c'est pas bon, (...) par contre, ça te met dans l'ambiance d'une équipe, de, d'un partage » (Marie).

Toutefois, par son caractère déculpabilisant, la commensalité pourrait bien affecter la relation entre PRN et comportement. "Quand on est entre amis ou en famille, (...) c'est normal de se faire plaisir. [La culpabilité], ça arrive plutôt (...) en rentrant un soir de la semaine, où voilà 
j'ai particulièrement faim, je me jette sur la nourriture, puis après je vais me dire, oh là là, j'ai trop mangé, c'était idiot» (Adeline). Même les aliments normalement bannis par l'individu deviennent temporairement acceptables lorsqu'il les partage : «tu pars en week-end avec les copains, et y a du Nutella pour les enfants, et tu te fais une bonne tartine: tu trouveras le Nutella exceptionnel, et t'auras raison d'en manger. (...) En solitaire, ce n'est plus un plaisir, non : après, tu culpabilises » (Marie). Pour les répondants, manger ne se pense donc pas uniquement en termes d'aliments ingurgités, mais aussi et peut-être surtout en termes de contextes de consommation, qui à leur tour semblent influencer la relation entre PRN et comportement.

Parmi les variables liées à l'environnement d'achat, l'hyperchoix

Une alimentation saine suppose une variété nutritionnelle. Mais cela rend les courses et les repas complexes, comme l'illustrent le tableau 4 et les verbatim en annexe A6.

[Tableau 4]

En effet, parce qu'il leur est difficile d'évaluer les caractéristiques nutritionnelles des aliments lorsqu'ils effectuent les courses, les répondants procèdent par comparaison : "c'est plus un truc relatif qu'absolu » (Thierry). Mais cela demande du temps et de l'énergie : «acheter pour faire une alimentation équilibrée et variée, (...) c'est un effort » (Adeline). La présence de produits en apparence semblables peut attirer leur attention sur un éventuel risque nutritionnel : «entre deux sauces tomate, d'un côté on peut avoir tomate, carottes, oignons, persil, et de l'autre côté, tomate, oignons, persil, dextrose, amidon modifié, blé reconstitué, ou sucre reconstitué, et on se dit mais pfff! " (Marie). "Ca peut m'arriver d'éplucher un peu [les étiquettes de pesto] pour me dire est-ce que, est-ce que c'est que de l'herbe, est-ce que c'est de l'herbe et de l'huile, est-ce que c'est de l'herbe, de l'huile et du fromage et encore autre chose quoi » (Thierry). Un choix abondant renforce encore le sentiment d'incertitude : « Notre peur, (...) c'est, devant l'opulence, être sûr d'avoir fait le bon choix. (...) C'est pas de manger, parce qu'on aura toujours à manger, (...) c'est d'avoir le choix » (Hervé). Or, l'incertitude est au cœur du risque perçu. L'hyperchoix pourrait donc augmenter la PRN.

D’ailleurs, les répondants adoptent des stratégies de réduction du choix, chez eux et sur le lieu de vente. «Dans notre frigo, on a rarement des choses très riches en calories » (Hervé). « J'ai juste une boîte de biscuits que je n'aime pas, pour si quelqu'un vient à l'improviste. (...) Donc chez moi, (...), sauf si je reçois, y a pas de Pépito, y a pas de Nutella. (...) Si y a, je vais dévorer. (...) Je suis obligée de me mettre des barrières " (Marie). Lorsqu’ils font leurs 
courses, face à l'abondance du choix, la grande majorité des répondants recourt à des réducteurs de risque nutritionnel. Certains sont intrinsèques, comme la naturalité perçue précédemment évoquée, d’autres sont extrinsèques. Ainsi, nombreux sont les répondants à mentionner le rôle du prix et de la marque dans leur recherche d'alimentation saine : «déjà, on ne prend jamais le prix vraiment discount » (Evelyne) ; "pour certains produits, je vais forcément acheter des marques en fait » (Laurence) ; «je vais me mettre en milieu de gamme, et après je vais regarder les différentes marques » (Adeline). Restreindre le choix semble alors un moyen de contrôler la PRN pour le consommateur.

\section{Les allégations nutritionnelles perturbatrices}

Quant aux allégations nutritionnelles, elles semblent augmenter la PRN auprès des répondants. Si la moitié des personnes interrogées y voit une information, mais à prendre avec du recul, l'autre moitié est plus critique (voir les verbatim en annexe A7) : "c'est de la pub ! (...). Il faudrait manger vingt yaourts par jour pendant des années, donc tout ça c'est pour faire vendre, c'est du mar-ke-ting » (Chantal). Globalement, les marques sont jugées incompétentes pour réaliser des allégations nutritionnelles : "pour avoir des études sérieuses (...), faut un recul de trente ou quarante ans, minimum. On est loin de les avoir » (JeanMarc). «Y a rien de prouvé, et la façon dont c'est libellé est toujours (...) un peu ambiguë» (Adeline). D’autre part, l’intégrité des gestionnaires de la marque est mise en doute. «Ils ont aucun intérêt à bien nous nourrir, ces gens-là. (...) Ils sont pas là pour nous faire du bien, (...) ils sont là pour développer leurs ventes » (Laurence). «La plupart du temps, quand ils mettent $0 \%$ de matière grasse, c'est de la publicité mensongère, parce que bon, c'est remplacé par de la matière grasse de synthèse ou des choses comme ça. Et c'est pas forcément bon pour la santé non plus » (Jérémy).

L'ajout d'allégations nutritionnelles pourrait même perturber l'image de la marque, d'après les verbatim liés à Danone, souvent cité : «Actimel, moi j’y crois pas à tout ça. Et pourtant, j'ai une belle-fille agro qui travaille chez Danone. Donc pour nous, Danone, tout était bon, mais je ne crois pas à ça » (Marie-Claude). «Je fais confiance au yaourt nature Danone. Je dirais que ça fait partie de mon patrimoine. Je n'imagine pas ne pas faire confiance à cette marque qui m'accompagne depuis toujours. (...) Mais je suis pas dupe quoi »(Marie). Il pourrait y avoir dissonance cognitive entre la dimension émotionnelle subjective de la marque et la démarche rationnelle objective des allégations nutritionnelles. Les marques en tant que telles peuvent constituer des réducteurs de PRN, mais le recours à des allégations semble 
perturber cette perception. L'ensemble de ces résultats ouvre des perspectives qui sont autant de propositions de recherche.

\section{DISCUSSION ET PISTES DE RECHERCHE SUR LA PRN}

Cette recherche qualitative fait ressortir l'importance de la PRN dans les comportements alimentaires, déjà identifiée dans la littérature. D’abord, tous les répondants lient l'alimentation à la santé. Cela rejoint les enquêtes nationales des Pouvoirs Publics, selon lesquelles $80 \%$ des Français estiment que l'alimentation a une influence sur la santé (Afsaa et Inpees, 2004). De plus, les femmes interviewées semblent plus inquiètes, conformément à la littérature (Bech-Larsen et Scholderer, 2007 ; Gomez, 2009b ; Grunert et Wills, 2007). Enfin, le risque nutritionnel prend le pas sur le risque sanitaire : ce n'est plus tant des produits avariés que les consommateurs ont peur, mais des effets secondaires à long terme de l'alimentation moderne (Fischler, 2001). En effet, aux yeux des répondants, le risque sanitaire est aujourd'hui mieux contrôlé et plus facilement maîtrisable, or le sentiment de contrôle réduit la perception de risque (Slovic, 1987). A l’inverse, les incertitudes entourant le risque nutritionnel sont amplifiées par la «cacophonie nutritionnelle » ambiante (IREMAS, 2010). Ses conséquences peu connues, diffuses, éloignées dans le temps, sont dès lors plus anxiogènes (Slovic et Peters, 2006). En outre, elles sont graves pour les répondants, a minima psychosociales, à l'extrême morbides voire vitales (Bergadaà et Urien, 2006 ; OMS et FAO, 2003). Or, l'incertitude et l'importance des conséquences du choix sont justement les deux dimensions classiques du risque perçu (Bauer, 1960 ; Cox, 1967 ; Cunningham, 1967 ; Mitchell, 1999 ; Taylor, 1974 ; Volle, 1995), encore renforcé quand les enjeux sont humains (Rosa, 2003 ; Slovic, 1987). Cela explique que les effets nutritionnels à long terme deviennent une vraie source d'inquiétude (Ascher, 2005 ; Lipovetsky, 2006 ; Mathé, Pilorin et Hébel, 2008 ; Pollan, 2009), et justifie l’intérêt d'étudier la PRN et ses mécanismes.

La naturalité perçue, une variable médiatrice de la relation entre allégations nutritionnelles et PRN?

Les personnes interviewées se méfient des allégations nutritionnelles, pourtant censées apporter une information sur la qualité des produits. Non seulement ces allégations ne les rassurent pas, mais elles soulèvent aussi des questions sur les aliments qui les portent. En ce sens, elles semblent augmenter plutôt que diminuer la PRN. Cela va à l'encontre des recherches anglo-saxonnes (Chandon et Wansink, 2010 ; Faulkner et al., 2013 ; Wansink et 
Chandon, 2006), mais à l'appui des recherches françaises (Fischler et al., 2010 ; Gurviez, 2010 ; Merdji, 2012). Les différences culturelles évoquées contribuent sans doute à l'expliquer, comme le soulignent les répondants eux-mêmes. En effet, le rationalisme des allégations nutritionnelles se heurte aux représentations sociales françaises de l'alimentation (Fischler et Masson, 2008 ; Gurviez, 2010).

Mais la recherche qualitative apporte une autre clé de lecture : les allégations nutritionnelles pourraient remettre en question la perception de naturalité du produit, étroitement associée à l’idée de bienfaits pour la santé. Les entretiens mettent clairement en avant une heuristique de naturalité. En effet, les répondants favorisent une liste simple d'ingrédients connus et une transformation minimale du produit, deux traits qui définissent la «préférence pour le naturel » (Rozin, 2005b ; Rozin et al., 2004). Des études comparatives montrent qu'elle est plus développée en France que dans les pays anglo-saxons (Fischler et Masson, 2008). D’après la recherche qualitative, les allégations nutritionnelles, la naturalité perçue et la PRN seraient toutes trois liées. Dès lors, il serait pertinent de vérifier la proposition suivante avec une démarche quantitative :

P1 : les allégations nutritionnelles supposant une transformation de l'aliment (de type « enrichi en» ou «allégé en ») diminuent la naturalité perçue du produit, ce qui augmente la perception de risque nutritionnel par le consommateur.

En outre, les entretiens menés confirment la différence d’appréciation entre allégations positives et négatives qui est mentionnée dans la littérature (Burton et Creyer, 2004 ; Moon et Balasubramanian, 2003 ; Verbeke et al., 2008). Plusieurs explications en sont données. D’abord, plus d'efforts de communication portent sur ce dont il faut limiter les apports, comme les matières grasses saturées, que sur ce qu'il faut manger en quantités suffisantes, comme le fer (Block et Peracchio, 2006). Ensuite, les informations négatives - par exemple limiter les matières grasses saturées pour réduire le cholestérol - ont plus d’impact que les informations positives - par exemple augmenter le fer pour améliorer le fonctionnement de l’organisme - (Moon et Balasubramanian, 2003 ; Siegrist, 2003 ; Verbeke et al., 2008). En effet, les consommateurs les comprennent mieux (Burton et Creyer, 2004). Enfin, il existe un « biais d’omission » (Rozin et al., 2004) : l’omission d’un nutriment est perçue comme plus naturelle que l'ajout, associé à une intervention humaine (Rozin, 2005a ; Rozin, 2006). Mais la présente étude va plus loin : l'ajout d'ingrédients semble susciter plus d'inquiétudes sur la qualité nutritionnelle du produit que le retrait. En effet, les verbatim posent la question de la 
congruence avec l'aliment. Cela conduit à envisager une influence du type d'allégations sur la naturalité perçue et donc sur la PRN :

P2 : les allégations de type «enrichi » diminuent plus la naturalité perçue du produit que celles de type « allégé », ce qui induit une perception du risque nutritionnel plus élevée.

La commensalité, une variable modératrice de la relation entre PRN et comportement ? D’après les entretiens, la PRN ne tient pas seulement aux caractéristiques du produit, mais aussi à la situation de consommation. Cela rejoint la théorie de Belk mettant en évidence l'influence de facteurs situationnels sur le comportement du consommateur (Belk, 1974; 1975). En particulier, la commensalité, partage de sa table (mensa) avec (cum) d’autres personnes (Fischler, 2011; Lardellier, 2011), semble réduire le stress inhérent à l'alimentation. Toutefois, d'après les réponses de l'étude qualitative, il convient de prendre en compte les contextes de consommation de façon plus détaillée, comme l’illustre la Figure 3.

[Figure 3]

Ce schéma met en avant la nécessité de distinguer les occasions festives des repas quotidiens (Sirieix, 1999). Lors d’occasions festives, les répondants peuvent s'autoriser en conscience des excès : la commensalité rend alors la PRN acceptable, ce qui semble favoriser la prise de risque nutritionnel. Au contraire, au quotidien, la commensalité permet de les alerter sur la PRN à cause du regard d'autrui, et ainsi de limiter leur prise de risque nutritionnel. La présence des tiers semble donc jouer un rôle complexe (de Castro, 2010).

Dans la littérature, les recherches se rejoignent sur le rôle normatif du groupe mais donnent des résultats a priori contradictoires. Herman et ses collègues l'expliquent par le fait que ces travaux se réfèrent à trois cadres théoriques différents (Herman, Roth et Polivy, 2003). (1) Ainsi, d'après la théorie de la facilitation sociale, la présence d'autres personnes augmente la prise alimentaire (Bell et Pliner, 2003 ; de Castro, 2010 ; Pliner et al., 2006 ; Stroebele et De Castro, 2004), à cause notamment d'un repas plus long qui fait perdre la notion du temps et des quantités (Wansink, 2006). (2) D’après la théorie de l'imitation, la commensalité encourage la consommation soit à la hausse soit à la baisse, selon le comportement des tiers modéré par la corpulence de ces derniers (McFerran et al., 2013). (3) Enfin, d’après la théorie de la gestion de l'image, la commensalité réduit la prise alimentaire lorsque les individus se sentent observés ou évalués (Herman, Roth et Polivy, 2003), ce qui explique pourquoi les femmes consomment moins de calories en présence d'hommes (Allen-O’Donnell et al., 2011 ; Mori, Chaiken et Pliner, 1987). 
Au vu de la littérature et de l'étude qualitative, le lien entre commensalité et comportement alimentaire ne semble donc pas direct. La commensalité jouerait un rôle modérateur sur la relation entre PRN et intention comportementale, différent selon le contexte festif ou non. Elle favoriserait une tempérance alimentaire au quotidien, mais pourrait au contraire encourager la prise de risque nutritionnel dans des contextes festifs, en désinhibant les individus face au risque nutritionnel perçu. D’où la proposition de recherche suivante, découpée en deux souspropositions :

P3a : dans un contexte quotidien, la PRN d'un individu qui prend un repas avec des convives induira un comportement alimentaire plus équilibré que si la personne prend son repas seule.

P3b : dans un contexte festif, la PRN d'un individu qui prend un repas avec des convives induira un comportement alimentaire moins équilibré que si la personne prend son repas seule.

\section{L'hyperchoix, une variable explicative de la PRN?}

L'offre alimentaire est aujourd'hui tellement large qu'il est impossible de maîtriser totalement, « aliment par aliment, ce qui est mangeable » (Apfelbaum, 1998). Cet hyperchoix pourrait accentuer la perception du risque nutritionnel pour l'alimentation en général, car la perte de contrôle est génératrice de risque perçu (Slovic, 1987). En effet, d’après les réponses des répondants, la nutrition réunit tous les prérequis à la situation d’hyperchoix identifiés dans la théorie (Scheibehenne, Greifeneder et Todd, 2010) : la difficulté à évaluer les différences nutritionnelles entre les produits (Larceneux, 2003b), le jugement par comparaison lié à la nécessité de choisir (Parker et Schrift, 2011), le recours à des heuristiques de décision (Gomez, 2009a), la pression temporelle (liée à l'exercice des courses). Certes, l’hyperchoix augmente la probabilité objective de trouver le produit idéal parmi l'assortiment, mais il en diminue aussi l'expérience subjective (Schwartz, 2004). Parce qu'il est coûteux en temps et en effort, il réduit la satisfaction du consommateur et sa motivation à choisir le produit (Iyengar et Lepper, 2000 ; Larceneux, Rieunier et Fady, 2007). Le « paradoxe du choix » peut éclairer l'absence de PRN relevée chez les deux répondantes qui ont connu la faim pendant la guerre, par «adaptation hédonique » (Schwartz, 2004). Ce processus psychologique permet à l'individu de s'habituer à une situation de plaisir ou de déplaisir, donc de supporter les privations. Mais dans les situations d’abondance, l'adaptation hédonique génère de l’insatisfaction, car le plaisir expérimenté finit par se révéler en deçà du plaisir anticipé 
(Schwartz, 2004). Cela pourrait expliquer pourquoi les Français, moins exposés à l’hyperchoix, semblent également moins stressés par l'alimentation que les Américains (Rozin et al., 2006).

Mais selon l'étude qualitative, l'environnement d'achat et notamment la taille de l'assortiment influencent aussi la PRN particulière à un aliment donné, ce qui constitue un résultat nouveau. D’après les verbatim, un même produit pourrait générer une PRN différente selon qu'il soit disposé dans un assortiment large ou étroit, car l'abondance de l’offre conduit les répondants à s’interroger sur les différences entre les produits. Cela les pousse notamment à comparer les compositions et à se poser des questions sur la qualité nutritionnelle, qu'ils n’auraient sans doute pas envisagées dans un contexte de choix réduit. D’où cette dernière proposition :

P4 : dans un contexte d'hyperchoix alimentaire pour un produit donné, la PRN de ce produit est plus élevée que dans un contexte de choix réduit.

\section{CONCLUSION}

S’appuyant sur une revue de littérature pluridisciplinaire et dix-huit entretiens en profondeur de consommateurs, cet article contribue à une meilleure compréhension des mécanismes de la Perception du Risque Nutritionnel. D’un point de vue théorique, il propose un nouveau cadre conceptuel de la PRN. En effet, la PRN, à travers ses deux dimensions de probabilité d’occurrence et de sévérité de la menace (Bauer, 1960), est un préalable à la modification des comportements alimentaires d'après le modèle de la motivation à se protéger (Rogers, 1975 ; Maddux et Rogers, 1983). La présente recherche qualitative permet d'envisager de nouvelles variables, influençant soit la PRN, soit le lien entre PRN et intention comportementale, comme illustré dans la Figure 4.

\section{[Figure 4]}

La naturalité perçue semble jouer un rôle de médiateur dans la relation entre allégations nutritionnelles et PRN. Les allégations nutritionnelles supposant une transformation de l'aliment (de type «enrichi en » ou «allégé en ») diminueraient la naturalité perçue des produits, ce qui à son tour augmenterait la PRN de ces aliments. La commensalité semble modérer la relation entre PRN et intention comportementale, de façon différente selon que le contexte soit festif (elle conduirait alors à une prise de risque nutritionnel accrue) ou quotidien (elle encouragerait alors un comportement alimentaire plus équilibré). Enfin, l’hyperchoix paraît accroître la PRN d'un aliment donné par rapport à un contexte de choix réduit. 
Certes, le recours à des photographies de plats traditionnels et de situations de consommation pour stimuler les répondants a pu exagérer l’importance de la naturalité et de la commensalité. Malgré cela, la naturalité est apparue spontanément chez les dix-huit répondants, et la commensalité chez treize d'entre eux, pendant la partie non directive des entretiens précédant les exercices projectifs. Des expérimentations seraient particulièrement appropriées pour vérifier l'existence des relations pressenties entre les variables, qui reposent ici sur des entretiens qualitatifs. De plus, comme souligné tout au long de la recherche, la composante culturelle est essentielle dans la PRN, ce qui signifie que ces résultats ne sont pas nécessairement valables en dehors d'un contexte français. Il serait intéressant d'étudier ces relations dans d'autres pays.

D’un point de vue managérial et social, cette recherche illustre la complexité des mécanismes du comportement alimentaire. En cela, elle remet en cause l'utilisation du modèle d'éducation dominant KABP - Knowledge, Attitudes, Beliefs and Practices - promu par l'Organisation Mondiale de la Santé (Calandre, Bricas et Sirieix, 2009 ; OMS, 1990). Ce modèle postule l'existence d'une relation linéaire entre la connaissance, l'attitude, les croyances et les pratiques en matière de santé. Il suffirait donc d'informer les populations pour in fine améliorer les comportements. Mais la réalité alimentaire est plus complexe (Calandre, Bricas et Sirieix, 2009), comme le montre la présente recherche. En effet, la PRN apparaît comme un des antécédents de l'intention comportementale, et elle-même semble influencée par de multiples facteurs, relatifs à l'aliment (les allégations nutritionnelles et la naturalité perçue), la situation de consommation (la commensalité), l'environnement d'achat (l'hyperchoix) ou l'individu (attitude face au risque nutritionnel). De plus, certaines de ces relations apparaissent directes, d'autres médiatrices, d'autres encore modératrices, d'où des interactions. Il ne suffit donc pas d'apporter l'information aux consommateurs pour qu'ils perçoivent «à bon escient » le risque nutritionnel et adaptent leur comportement.

Quelles sont les implications de ces résultats pour les pouvoirs publics ? D’après la présente recherche, la naturalité perçue diminue la PRN. Or, l'industrie agroalimentaire en est consciente. Un article de presse professionnelle récent ${ }^{4}$ recommande aux entreprises d'utiliser « un des nombreux autres moyens de communiquer la naturalité sans utiliser le mot 'naturel' (...) pour ne pas s'enliser dans un champ de mine réglementaire », et pour donner aux produits un «halo santé [qui] augmentera les ventes de façon significative ». Pour éviter les

\footnotetext{
${ }^{4}$ http://www.foodmanufacture.co.uk/Ingredients/Top-12-trends-for-food-nutrition-and-health, paru le
} 18 décembre 2013 
tromperies, il apparaît donc nécessaire de lutter contre les signaux qui suggéreraient une naturalité non avérée, surtout si les produits ont un profil nutritionnel contestable. En termes d'hyperchoix, les pouvoirs publics peuvent faciliter le choix « vertueux » des consommateurs par un étiquetage clair, lisible et bien compris. Ils pourraient également communiquer sur ce que la revue de la littérature a mis en évidence : un produit «light » n’autorise pas le consommateur soucieux de sa santé à en consommer à forte dose. Ceci, comme pour les cigarettes, n’est pas forcément connu de la population française. Enfin, concernant la commensalité, les pouvoirs publics devraient inciter les individus à prendre des repas quotidiens en famille, et ne pas focaliser leur communication uniquement sur les nutriments comme c'est encore souvent le cas dans le monde (Bublitz et al., 2013 ; Fischler et Masson, 2008). En France, les dernières versions du Programme National Nutrition Santé (PNNS) et du Programme National pour l'Alimentation (PNA) mentionnent désormais les « dimensions symboliques et sociales de l'acte alimentaire » (PNNS) et la « convivialité des repas » (PNA). C’est aussi ce que préconise un courant de recherche récent, le paradigme du bien-être alimentaire, défini comme une «relation individuelle et sociétale positive à l'alimentation, d'un point de vue psychologique, physique, émotionnel et social » (Block et al., 2011 ; Bublitz et al., 2013). Cette approche holiste de l'alimentation, laissant la place aux dimensions hédonique et sociale, paraît tout à fait appropriée compte-tenu de la présente recherche. 
ANNEXE A1 : Synthèse d'articles publiés dans des revues ou ouvrages majeurs sur le lien entre allégations nutritionnelles et PRN

\begin{tabular}{|c|c|c|c|}
\hline Références & Objectif principal & Questions posées et méthodologie & Principaux résultats pour la présente recherche \\
\hline $\begin{array}{l}\text { (Andrews, } \\
\text { Netemeyer et } \\
\text { Burton, 1998) }\end{array}$ & $\begin{array}{l}\text { Mettre en évidence } \\
\text { l'effet de halo santé des } \\
\text { allégations } \\
\text { nutritionnelles }\end{array}$ & $\begin{array}{l}\text { Les consommateurs comprennent-ils les allégations } \\
\text { nutritionnelles? Généralisent-ils les bénéfices } \\
\text { nutritionnels à d'autres nutriments? } \\
\text { Expérimentations inter-sujets, } 365 \text { consommateurs }\end{array}$ & $\begin{array}{l}\text { Cette recherche est la première à mettre en évidence le halo santé : en } \\
\text { présence d'allégations nutritionnelles, les consommateurs infèrent des } \\
\text { qualités nutritionnelles supérieures aussi pour les autres nutriments non } \\
\text { mentionnés. }\end{array}$ \\
\hline $\begin{array}{l}\text { (Burton et } \\
\text { Creyer, 2004) }\end{array}$ & $\begin{array}{l}\text { Evaluer l'influence d'une } \\
\text { information } \\
\text { nutritionnelle apposée } \\
\text { sur les cartes des } \\
\text { restaurants }\end{array}$ & $\begin{array}{l}\text { Comment l’information nutritionnelle, les } \\
\text { allégations santé, et la présentation influencent-ils } \\
\text { le jugement des consommateurs? } \\
\text { Expérimentation inter-sujets, } 377 \text { répondants. }\end{array}$ & $\begin{array}{l}\text { Les consommateurs accordent plus de crédibilité aux informations } \\
\text { nutritionnelles qu'aux allégations santé, mais les allégations santé } \\
\text { influencent la perception et la crédibilité selon le contexte. } \\
\text { Les consommateurs interprètent plus facilement l'information négative } \\
\text { que l'information positive. }\end{array}$ \\
\hline $\begin{array}{l}\text { (Chandon et } \\
\text { Ordabayeva, } \\
\text { 2009) }\end{array}$ & $\begin{array}{l}\text { Mesurer les effets des } \\
\text { changements de } \\
\text { dimensions spatiales sur } \\
\text { les quantités perçues }\end{array}$ & $\begin{array}{l}\text { Comment les individus estiment-ils les } \\
\text { changements de taille de portion ou d'emballage ? } \\
\text { Comment cela influence-t-il leur consommation et } \\
\text { leurs décisions d'achat? } \\
5 \text { expérimentations, centaines de personnes }\end{array}$ & $\begin{array}{l}\text { Plus il y a de dimensions, plus les consommateurs sous-estiment la } \\
\text { quantité contenue et la magnitude des changements, ce qui conduit à une } \\
\text { surconsommation. Cette recherche met en évidence les biais de taille et } \\
\text { de format. }\end{array}$ \\
\hline $\begin{array}{l}\text { (Chandon et } \\
\text { Wansink, } \\
\text { 2007a) }\end{array}$ & $\begin{array}{l}\text { Bâtir un modèle } \\
\text { psychophysique de } \\
\text { l'évaluation du nombre } \\
\text { de calories dans un repas }\end{array}$ & $\begin{array}{l}\text { Les personnes en surpoids sous-évaluent-elles plus } \\
\text { la teneur énergétique de leur repas que les } \\
\text { personnes de poids normal et pourquoi ? Comment } \\
\text { atténuer les biais d'évaluation ? Qu'en est-il des } \\
\text { diététiciens ? } \\
4 \text { études : } 55 \text { puis } 156 \text { étudiants en laboratoire, } 147 \\
\text { adultes sur le terrain, } 405 \text { diététiciens en laboratoire }\end{array}$ & $\begin{array}{l}\text { Plus le nombre de calories est élevé, plus il est sous-estimé ; moins il est } \\
\text { élevé, plus il est surestimé, quels que soient l’IMC et l'implication } \\
\text { nutritionnelle. Les personnes en surpoids sous-estiment plus le nombre } \\
\text { de calories de leur repas que les personnes de poids normal mais } \\
\text { seulement parce qu’elles mangent de plus grandes quantités : le biais se } \\
\text { manifeste plus. Avertir les individus de ce biais ne suffit pas à régler le } \\
\text { problème. En revanche, décomposer les calories par plat permet de } \\
\text { mieux évaluer le nombre de calories total pour les grandes quantités. } \\
\text { Le même biais est noté chez les diététiciens, mais moins marqué. }\end{array}$ \\
\hline $\begin{array}{l}\text { (Chandon et } \\
\text { Wansink, } \\
\text { 2007b) }\end{array}$ & $\begin{array}{l}\text { Quel est l'effet du } \\
\text { positionnement santé de } \\
\text { certains fast-foods sur la } \\
\text { consommation? }\end{array}$ & $\begin{array}{l}\text { Existe-t-il un effet de halo santé dans les fast } \\
\text { foods ? Comment le réduire ? } \\
4 \text { expérimentations : } 1 \text { sur le terrain ( } 518 \\
\text { répondants chez McDonald ou Subway), } 3 \text { en } \\
\text { laboratoire (316, 46, } 214 \text { étudiants) }\end{array}$ & $\begin{array}{l}\text { Les allégations santé conduisent les consommateurs à sous-estimer le } \\
\text { nombre de calories des plats principaux dans les fast-foods et choisir des } \\
\text { accompagnements et boissons plus riches. Les consommateurs ne sont } \\
\text { pas conscients de ces biais. Développer l'esprit critique des } \\
\text { consommateurs envers les allégations nutritionnelles réduit l'effet de } \\
\text { halo santé. }\end{array}$ \\
\hline
\end{tabular}




\begin{tabular}{|c|c|c|c|c|}
\hline $\begin{array}{l}\text { (Chandon et } \\
\text { Wansink, 2010) }\end{array}$ & $\begin{array}{l}\text { Comprendre le lien entre } \\
\text { le marketing alimentaire } \\
\text { et l’obésité }\end{array}$ & $\begin{array}{l}\text { Comment chacun des éléments du mix-marketing } \\
\text { influence-t-il la consommation alimentaire? } \\
\text { Revue de littérature pluridisciplinaire }\end{array}$ & \multicolumn{2}{|c|}{$\begin{array}{l}\text { Tous les éléments du mix-marketing influencent la consommation } \\
\text { alimentaire : le prix (long terme et promotionnel), la communication } \\
\text { marketing (publicité, promotion, marque, allégations nutritionnelles et } \\
\text { santé), le produit (qualités sensorielles et nutritionnelles, taille et forme } \\
\text { de l'emballage et des portions), l'environnement de consommation } \\
\text { (accès, visibilité, praticité, taille et forme des couverts, ambiance). }\end{array}$} \\
\hline $\begin{array}{l}\text { (Chandon, } \\
\text { 2012) }\end{array}$ & $\begin{array}{l}\text { Comprendre le lien entre } \\
\text { packaging et obésité }\end{array}$ & $\begin{array}{l}\text { Comment les différents aspects du packaging } \\
\text { influencent-ils la consommation alimentaire : taille, } \\
\text { forme, allégations, branding... ? } \\
\text { Revue de littérature }\end{array}$ & \multicolumn{2}{|c|}{$\begin{array}{l}\text { Tous les aspects du packaging (taille, forme, allégations nutritionnelles, } \\
\text { branding...) influencent la perception du consommateur, généralement à } \\
\text { son insu. Les recherches montrent l'existence de nombreux biais de } \\
\text { perception et un effet de halo santé. }\end{array}$} \\
\hline $\begin{array}{l}\text { (Chernev et } \\
\text { Gal, 2010) }\end{array}$ & $\begin{array}{l}\text { Comprendre comment } \\
\text { les consommateurs } \\
\text { évaluent les options } \\
\text { alimentaires en cas de } \\
\text { conflit plaisir / santé }\end{array}$ & $\begin{array}{l}\text { Comment les consommateurs évaluent-ils les } \\
\text { teneurs en calories d'aliments « mauvais » vs } \\
\text { « vertueux » vs combinaison des deux? } \\
4 \text { expérimentations, plusieurs centaines d'individus }\end{array}$ & \multicolumn{2}{|c|}{$\begin{array}{l}\text { Au-delà de l'effet de halo santé, il existe un biais de moyenne. Les } \\
\text { combinaisons d'aliments conduisent à une sous-estimation des calories : } \\
\text { les répondants attribuent moins de calories à un hamburger + salade vs } \\
\text { hamburger seul, car ils font la moyenne des calories. Cela est dû à une } \\
\text { catégorisation bon / mauvais pour la santé, sans considérer les quantités. }\end{array}$} \\
\hline $\begin{array}{l}\text { (Faulkner et al., } \\
\text { 2013) }\end{array}$ & $\begin{array}{l}\text { Evaluer l'influence de la } \\
\text { perception santé des } \\
\text { aliments sur leur } \\
\text { consommation }\end{array}$ & $\begin{array}{l}\text { Comment les allégations nutritionnelles suggérant } \\
\text { un aliment plus sain influencent-elles la densité } \\
\text { énergétique perçue, la taille des portions } \\
\text { consommées et la culpabilité anticipée à l’idée de } \\
\text { manger le produit? } \\
\text { Recherche médicale, expérimentations sur } 186 \\
\text { Irlandais du Nord }\end{array}$ & \multicolumn{2}{|c|}{$\begin{array}{l}\text { Les consommateurs estiment à tort que les aliments positionnés santé } \\
\text { sont moins énergétiques que les aliments standard. Ils sous-estiment la } \\
\text { taille des portions recommandées, surtout pour les produits positionnés } \\
\text { comme sains, ce qui conduit à une prise de calories accrue. La } \\
\text { culpabilité anticipée est moindre pour les versions santé vs standard. Les } \\
\text { allégations nutritionnelles pourraient donc conduire à des } \\
\text { comportements alimentaires non souhaitables. }\end{array}$} \\
\hline \multirow[t]{9}{*}{$\begin{array}{l}\text { (Fischler et al., } \\
\text { 2010) }\end{array}$} & \multirow{9}{*}{$\begin{array}{l}\text { Etude des représentations } \\
\text { du bien-manger en } \\
\text { Europe, et de la } \\
\text { perception des } \\
\text { allégations } \\
\text { nutritionnelles des } \\
\text { consommateurs } \\
\text { européens suite au } \\
\text { règlement européen } \\
\text { 1924/2006 }\end{array}$} & \multirow{9}{*}{$\begin{array}{l}\text { Comment les allégations nutritionnelles sont-elles } \\
\text { perçues par les consommateurs européens? } \\
\text { En termes marketing, sont-elles réellement une } \\
\text { source de valeur pour l’industriel ? } \\
\text { Etude en souscription menée en partenariat avec les } \\
\text { instituts Protéines et BrainValue pour des } \\
\text { industries agro-alimentaires en } 2010 \text { : recherche } \\
\text { qualitative ( } 2 \text { focus-groups / pays) et quantitative } \\
\text { (1000 personnes / pays) dans six pays européens : } \\
\text { la France, le Royaume-Uni, la Suisse, l'Allemagne, } \\
\text { la Pologne et la Suède }\end{array}$} & \multicolumn{2}{|c|}{$\begin{array}{l}\text { Les allégations nutritionnelles suscitent des suspicions chez les } \\
\text { Européens, surtout les Français. Valeur santé de } 1 \text { (faible) à } 5 \text { (fort) } \\
\text { attribuée par les Français aux yaourts : }\end{array}$} \\
\hline & & & \begin{tabular}{|l|} 
Yaourt sans additifs ni conservateurs \\
\end{tabular} & 4,03 \\
\hline & & & Yaourt fait à la maison & 4,03 \\
\hline & & & Yaourt aux fruits frais & 3,82 \\
\hline & & & Yaourt bio & 3,53 \\
\hline & & & Yaourt avec ferment utile à la digestion & 3,44 \\
\hline & & & Yaourt avec conservateurs naturels & 3,47 \\
\hline & & & Yaourt avec ingrédient bénéfique santé & 3,28 \\
\hline & & & Yaourt allégé en matière grasse & 3,22 \\
\hline
\end{tabular}




\begin{tabular}{|c|c|c|c|}
\hline $\begin{array}{l}\text { (Garg, Wansink } \\
\text { et Inman, 2007) }\end{array}$ & $\begin{array}{l}\text { Comprendre comment } \\
\text { les émotions influencent } \\
\text { la prise alimentaire }\end{array}$ & $\begin{array}{l}\text { Comment les émotions passagères (tristesse, joie) } \\
\text { influencent-elles la consommation de produits } \\
\text { hédoniques vs non hédoniques ? L'information } \\
\text { nutritionnelle modère-t-elle ces relations? } \\
3 \text { expérimentations auprès d'étudiants, } \\
\text { manipulation de l'humeur par des histoires (films } \\
\text { ou textes) }\end{array}$ & $\begin{array}{l}\text { En l'absence d'informations nutritionnelles négatives, les personnes } \\
\text { consomment 30\% de plus de produits hédoniques (popcorns, M\&Ms) } \\
\text { en état de tristesse qu'en état de joie. Au contraire, ils mangent de plus } \\
\text { grandes quantités de produits non hédoniques (raisins secs) en état de } \\
\text { joie qu'en état de tristesse. La présence d'informations nutritionnelles } \\
\text { négatives modère ces effets chez les personnes tristes mais pas chez les } \\
\text { personnes joyeuses. Il n’y a pas d'effet de genre. }\end{array}$ \\
\hline $\begin{array}{l}\text { (Garretson et } \\
\text { Burton, 2000) }\end{array}$ & $\begin{array}{l}\text { Mesurer l'influence de la } \\
\text { nouvelle présentation des } \\
\text { valeurs nutritionnelles } \\
\text { américaine NLEA et des } \\
\text { allégations } \\
\text { nutritionnelles et de santé } \\
\text { sur le jugement des } \\
\text { consommateurs }\end{array}$ & $\begin{array}{l}\text { Un niveau de lipides élevé dans la table } \\
\text { nutritionnelle modifie-t-il le jugement des } \\
\text { consommateurs ? Quid des fibres ? Quid des } \\
\text { allégations nutritionnelles? } \\
\text { Expérimentations intra-sujets, } 382 \text { personnes, } \\
\text { évaluation de packagings, 3x5 conditions de lipides } \\
\text { et fibres (table nutritionnelle / allégations). }\end{array}$ & $\begin{array}{l}\text { L'allégation faible teneur en matière grasse n'exerce pas d'influence sur } \\
\text { le jugement du consommateur (attitude envers la marque, envers le } \\
\text { niveau nutritionnel et intention d'achat), contrairement à un chiffre } \\
\text { élevé dans la table nutritionnelle. Pour les fibres, ni l'allégation, ni le } \\
\text { niveau dans la table nutritionnelle ne modifient le jugement du } \\
\text { consommateur. En cas d’incongruence perçue, les consommateurs } \\
\text { accordent la primeur aux tables nutritionnelles et leur confiance en } \\
\text { l'allégation est affectée. }\end{array}$ \\
\hline $\begin{array}{l}\text { (Keller et al., } \\
\text { 1997) }\end{array}$ & $\begin{array}{l}\text { Evaluer l'effet des } \\
\text { nouvelles tables } \\
\text { nutritionnelles } \\
\text { américaines (NLEA) sur } \\
\text { la perception des } \\
\text { consommateurs }\end{array}$ & $\begin{array}{l}\text { Quels sont les effets des allégations santé, des } \\
\text { tables nutritionnelles et de la motivation sur les } \\
\text { évaluations des consommateurs depuis la mise en } \\
\text { place des NLEA en } 1994 \text { ? } \\
\text { Expérimentation inter-sujets, } 460 \text { personnes. }\end{array}$ & $\begin{array}{l}\text { Les consommateurs font plus confiance aux valeurs nutritionnelles } \\
\text { qu'aux allégations : les valeurs nutritionnelles sont plus utiles au choix, } \\
\text { alors que les allégations, perçues comme du marketing, génèrent de la } \\
\text { suspicion. La présence d'allégations n’améliore pas la perception du } \\
\text { consommateur ni ses intentions d'achat Si elles sont incongruentes avec } \\
\text { les valeurs nutritionnelles, elles nuisent à la crédibilité du fabricant. }\end{array}$ \\
\hline $\begin{array}{l}\text { (Kozup, Creyer } \\
\text { et Burton, 2003) }\end{array}$ & $\begin{array}{l}\text { Evaluer l'influence de } \\
\text { l'information } \\
\text { nutritionnelle et } \\
\text { d’allégations santé } \\
\text { placées sur des menus de } \\
\text { restaurant et des } \\
\text { emballages de produits }\end{array}$ & $\begin{array}{l}\text { Quelle est l'influence de l’allégation santé vs les } \\
\text { informations nutritionnelles sur l'évaluation du } \\
\text { consommateur et le risque de maladie perçu? } \\
3 \text { expérimentations intra-sujets, lasagnes vendues } \\
\text { en supermarché ou sur une carte de restaurant. }\end{array}$ & $\begin{array}{l}\text { Les informations nutritionnelles influencent plus l'évaluation du } \\
\text { consommateur (attitude, intention d'achat) que l'allégation santé. Les } \\
\text { consommateurs accordent plus d'importance aux informations } \\
\text { nutritionnelles qu'aux allégations. Dans le contexte des restaurants, les } \\
\text { consommateurs réagissent plus fortement à des informations négatives. } \\
\text { Si celles des autres produits sont plus négatives que celles du produit } \\
\text { cible, les attitudes et intentions d'achat du produit cible augmentent. }\end{array}$ \\
\hline $\begin{array}{l}\text { (Lone et al., } \\
\text { 2009) }\end{array}$ & $\begin{array}{l}\text { Marketer une } \\
\text { alimentation saine auprès } \\
\text { des consommateurs les } \\
\text { moins intéressés }\end{array}$ & $\begin{array}{l}\text { Comment promouvoir une alimentation plus saine } \\
\text { auprès des consommateurs qui se désintéressent de } \\
\text { la nutrition? } \\
\text { Questionnaires auprès de } 520 \text { étudiants californiens }\end{array}$ & $\begin{array}{l}\text { Il ne vaut mieux pas mettre en évidence de messages nutritionnels } \\
\text { auprès des hommes car cela les détourne des produits, contrairement } \\
\text { aux femmes. }\end{array}$ \\
\hline
\end{tabular}




\begin{tabular}{|c|c|c|c|c|c|}
\hline $\begin{array}{l}\text { (McCann et al., } \\
\text { 2013) }\end{array}$ & $\begin{array}{l}\text { Evaluer l'influence des } \\
\text { allégations } \\
\text { nutritionnelles sur la } \\
\text { taille des portions } \\
\text { consommées }\end{array}$ & $\begin{array}{l}\text { Comment les allégations nutritionnelles de type } \\
\text { allégé influencent-elles la quantité consommée ? } \\
\text { Recherche médicale, expérimentations auprès de } \\
47 \text { Irlandais du Nord, } 3 \text { repas identiques mais } \\
\text { informations standard vs haute teneur en graisse et } \\
\text { calories vs faible teneur en graisse et calories }\end{array}$ & \multicolumn{3}{|c|}{$\begin{array}{l}\text { La quantité consommée et la prise énergétique sont significativement } \\
\text { augmentées dans la condition faible teneur en graisse et calories, mais } \\
\text { identiques entre les conditions standard et haute teneur en graisse et } \\
\text { calories. Les individus qui augmentent le plus les quantités consommées } \\
\text { dans la condition faible teneur en graisse et calories sont les hommes et } \\
\text { les personnes en surpoids. Cela suggère que les allégations } \\
\text { nutritionnelles de type allégé sont un facteur de surconsommation. }\end{array}$} \\
\hline \multirow[t]{8}{*}{$\begin{array}{l}\text { (Merdji, } 2012 \text {; } \\
\text { Merdji, 2010) }\end{array}$} & \multirow{8}{*}{$\begin{array}{l}\text { Etude des effets du } \\
\text { règlement européen } \\
\text { 1924/2006 : influence } \\
\text { des allégations } \\
\text { nutritionnelles sur la } \\
\text { perception de santé et de } \\
\text { naturalité des } \\
\text { consommateurs français }\end{array}$} & \multirow{8}{*}{$\begin{array}{l}\text { Quelle valeur santé et quelle valeur naturalité les } \\
\text { consommateurs attribuent-ils à des aliments selon } \\
\text { les allégations qu'ils portent? } \\
\text { Enquête ANR réalisée par M Merdji, G Debucquet } \\
\text { et C Fischler auprès de } 1000 \text { adultes français en } \\
\text { décembre } 2009 \text { : combinaison d'entretiens, de } \\
\text { questionnaires et de méthodes d'économie } \\
\text { expérimentale (enchères), pour plusieurs catégories } \\
\text { de produits }\end{array}$} & \multicolumn{3}{|c|}{$\begin{array}{l}\text { Il existe un décalage entre la valeur santé exprimée par les allégations et } \\
\text { celle perçue par les consommateurs français. } \\
\text { Ex du jambon, notes de } 1 \text { (faible) à } 10 \text { (forte) : }\end{array}$} \\
\hline & & & Type de jambon & Valeur santé & Valeur naturalité \\
\hline & & & Artisanal & 7.27 & 7.49 \\
\hline & & & Bio & 6.47 & 7.11 \\
\hline & & & Standard & 5.40 & 5.54 \\
\hline & & & Allégé en gras & 5.19 & 4.45 \\
\hline & & & Ajout omégas 3 & 4.15 & 3.43 \\
\hline & & & \multicolumn{3}{|c|}{$\begin{array}{l}\text { Les Français sont hostiles à l'égard des produits enrichis. Les produits } \\
\text { laitiers, les céréales du petit-déjeuner (consommation individuelle), les } \\
\text { corps gras (ingrédients) se prêtent mieux aux allégations. }\end{array}$} \\
\hline $\begin{array}{l}\text { (Mishra, Mishra } \\
\text { et Masters, } \\
\text { 2012) }\end{array}$ & $\begin{array}{l}\text { Etudier l’influence de la } \\
\text { taille des bouchées sur la } \\
\text { quantité consommée }\end{array}$ & $\begin{array}{l}\text { Pourquoi et comment la taille des bouchées } \\
\text { influence-t-elle la quantité mangée? } \\
\text { Expérimentation dans un restaurant puis en } \\
\text { laboratoire. Manipulation de la taille des bouchées } \\
\text { par la taille de la fourchette mise à disposition }\end{array}$ & \multicolumn{3}{|c|}{$\begin{array}{l}\text { Des bouchées plus petites augmentent la quantité consommée au } \\
\text { restaurant. La théorie de la motivation en fournit l'explication : au } \\
\text { restaurant, l'objectif est d'être rassasié et d'en avoir pour son argent. } \\
\text { L’étude en laboratoire montre que quand ces facteurs sont absents, le } \\
\text { résultat est inverse. }\end{array}$} \\
\hline $\begin{array}{l}\text { (Rozin, } \\
\text { Millman et } \\
\text { Nemeroff, } \\
1986)\end{array}$ & $\begin{array}{l}\text { Mesurer l’application de } \\
\text { la pensée magique } \\
\text { (Frazer, Mauss) dans la } \\
\text { perception alimentaire }\end{array}$ & $\begin{array}{l}\text { Quels sont les effets de contagion et de similarité, } \\
\text { en positif et en négatif, sur la perception des } \\
\text { individus? } \\
\text { Série d'expériences auprès de } 50 \text { personnes }\end{array}$ & \multicolumn{3}{|c|}{$\begin{array}{l}\text { Ces expériences mettent en évidence deux biais de perception : le } \\
\text { principe de contagion et le principe de similarité. Il existe une } \\
\text { asymétrie : la contagion et la similarité sont plus fortes en négatif qu’en } \\
\text { positif. }\end{array}$} \\
\hline $\begin{array}{l}\text { (Saulais et al., } \\
\text { 2012) }\end{array}$ & $\begin{array}{l}\text { Comparer la relation } \\
\text { entre connaissances } \\
\text { nutritionnelles et choix } \\
\text { alimentaires dans } 3 \text { pays }\end{array}$ & $\begin{array}{l}\text { Quelles sont les connaissances nutritionnelles des } \\
\text { consommateurs sur les matières grasses aux Etats- } \\
\text { Unis vs au Québec vs en France? } \\
\text { Questionnaires, environ } 100 \text { personnes / pays }\end{array}$ & \multicolumn{3}{|c|}{$\begin{array}{l}\text { Un nouveau paradoxe français ? Les consommateurs américains ont } \\
\text { beaucoup plus de connaissances nutritionnelles sur les matières grasses } \\
\text { que les Français, et pourtant le taux d’obésité est trois fois supérieur aux } \\
\text { Etats-Unis qu'en France. }\end{array}$} \\
\hline
\end{tabular}




\begin{tabular}{|c|c|c|c|}
\hline $\begin{array}{l}\text { (Schuldt, } \\
\text { Muller et } \\
\text { Schwarz, 2012) }\end{array}$ & $\begin{array}{l}\text { Mesurer l'effet des } \\
\text { allégations commerce } \\
\text { équitable sur la PRN }\end{array}$ & $\begin{array}{l}\text { Les allégations liées au commerce équitables } \\
\text { produisent-elles un effet de halo santé ? } \\
2 \text { expérimentations ( } 56 \text { adultes, } 192 \text { étudiants) }\end{array}$ & $\begin{array}{l}\text { Un chocolat étiqueté commerce équitable est perçu comme contenant } \\
\text { moins de calories. Cette inférence existe même sans la présence } \\
\text { d'allégations, si l'entreprise est décrite comme traitant bien ses salariés. }\end{array}$ \\
\hline $\begin{array}{l}\text { (Sharpe, Staelin } \\
\text { et Huber, 2008) }\end{array}$ & $\begin{array}{l}\text { Monter l'intérêt d’utiliser } \\
\text { l'aversion aux extrêmes } \\
\text { pour lutter contre } \\
\text { l'obésité }\end{array}$ & $\begin{array}{l}\text { Comment la gamme de formats de sodas proposée } \\
\text { dans les fast-foods influence-t-elle le choix du } \\
\text { consommateur et sa prise énergétique? } \\
3 \text { expériences intra-sujets, scénarios et } \\
\text { environnement réel, plusieurs centaines d’adultes }\end{array}$ & $\begin{array}{l}\text { Les consommateurs sont averses aux extrêmes : ajouter un format plus } \\
\text { grand à la gamme proposée ou retirer le plus petit augmente les } \\
\text { consommations individuelles. Il faut prendre en compte le référentiel } \\
\text { dans les politiques publiques : pour réduire la prise de calories, ajouter } \\
\text { un format plus petit est efficace car cela conduit les consommateurs à } \\
\text { choisir des portions plus petites. }\end{array}$ \\
\hline $\begin{array}{l}\text { (Verbeke et alii, } \\
\text { 2008) }\end{array}$ & $\begin{array}{l}\text { Estimer l'influence d'une } \\
\text { communication portant } \\
\text { sur les risques et/ou } \\
\text { bénéfices nutritionnels } \\
\text { sur la consommation et } \\
\text { la perception des } \\
\text { attributs du poisson en } \\
\text { Belgique }\end{array}$ & $\begin{array}{l}\text { Comment le consommateur traite-t-il les aspects } \\
\text { positifs (oméga } 3 \text { et vitamine D) et négatifs } \\
\text { (dioxines et mercure) de la communication } \\
\text { nutritionnelle ? Quelle est l’influence de la source } \\
\text { d'information et de la crédibilité perçue? } \\
\text { Expérimentation sur } 381 \text { femmes }\end{array}$ & $\begin{array}{l}\text { La condition «bénéfices » augmente l'intention de consommation de } \\
21 \% \text { mais ne change pas fondamentalement la perception des attributs, } \\
\text { déjà très positive (effet de plafond). La condition « risques » diminue } \\
\text { fortement la perception des attributs mais moins les intentions de } \\
\text { consommation (de } 8 \% \text { ) car le message est jugé peu crédible et peu } \\
\text { digne de confiance. Les conditions « bénéfices puis risques » et } \\
\text { « risques puis bénéfices » diminuent la perception des attributs mais ne } \\
\text { changent pas significativement l'intention de consommation. }\end{array}$ \\
\hline $\begin{array}{l}\text { (Wansink et } \\
\text { Chandon, 2006) }\end{array}$ & $\begin{array}{l}\text { Evaluer l’influence de } \\
\text { l'allégation « faible } \\
\text { teneur en matières } \\
\text { grasses » sur la } \\
\text { consommation }\end{array}$ & $\begin{array}{l}\text { Les allégations « faible teneur en matières } \\
\text { grasses » augmentent-elles la consommation ? Quel } \\
\text { rôle jouent le sentiment de culpabilité et la taille } \\
\text { des portions estimée ? L’indication de taille des } \\
\text { portions peut-elle éliminer ces biais potentiels? } \\
3 \text { expérimentations, plusieurs centaines d'adultes }\end{array}$ & $\begin{array}{l}\text { Les participants mangent à leur insu jusqu’à 50\% de plus quand les } \\
\text { aliments sont présentés comme allégés en matières grasses. Chez les } \\
\text { personnes de poids normal, cela vaut surtout pour les produits « sain » } \\
\text { (Granola). Chez les personnes en surpoids, cela concerne les produits } \\
\text { hédoniques et sains, et dans des proportions encore plus élevées. En } \\
\text { effet, l'allégation diminue le sentiment de culpabilité et conduit à sous- } \\
\text { estimer les calories contenues, donc à surévaluer la portion appropriée. }\end{array}$ \\
\hline $\begin{array}{l}\text { (Werle et al., } \\
\text { 2012) }\end{array}$ & $\begin{array}{l}\text { Améliorer l'efficacité } \\
\text { des campagnes de } \\
\text { prévention de l'obésité } \\
\text { auprès d'adolescents }\end{array}$ & $\begin{array}{l}\text { Quelle est l’efficacité de l’argument social vs santé } \\
\text { auprès des adolescents dans la lutte contre } \\
\text { l'obésité ? } \\
\text { Expérimentation auprès de } 797 \text { jeunes défavorisés }\end{array}$ & $\begin{array}{l}\text { L’argument social est plus efficace que l'argument santé pour améliorer } \\
\text { les choix alimentaires des adolescents. }\end{array}$ \\
\hline $\begin{array}{l}\text { (Wilcox et al., } \\
\text { 2009) }\end{array}$ & $\begin{array}{l}\text { Etudier l'effet du } \\
\text { contrôle personnel sur le } \\
\text { comportement } \\
\text { alimentaire }\end{array}$ & $\begin{array}{l}\text { Comment la présence d’aliments plus sains } \\
\text { influence-t-elle le choix des individus selon leur } \\
\text { niveau de contrôle personnel ? } \\
4 \text { expérimentations, plusieurs centaines d'étudiants }\end{array}$ & $\begin{array}{l}\text { Ironiquement, la simple présence d’un aliment plus sain dans un } \\
\text { assortiment par ailleurs peu sain conduit les individus à fort contrôle } \\
\text { personnel à choisir les aliments les moins sains. }\end{array}$ \\
\hline
\end{tabular}


ANNEXE A2 : Profil des personnes interviewées

\begin{tabular}{|c|c|c|c|c|c|c|c|}
\hline Prénom & Sexe & $\begin{array}{l}\text { Age } \\
\text { (ans) }\end{array}$ & Lieu & $\begin{array}{c}\text { Type } \\
\text { d'habitat }\end{array}$ & $\begin{array}{l}\text { Situation } \\
\text { familiale }\end{array}$ & $\begin{array}{l}\text { Niveau } \\
\text { d'étude }\end{array}$ & Emploi \\
\hline Adeline & $\mathrm{F}$ & 38 & $\begin{array}{l}\text { Banlieue } \\
\text { parisienne }\end{array}$ & Appartement & $\begin{array}{l}\text { Mariée } \\
\text { Enfants }\end{array}$ & $\mathrm{Bac}+5$ & Cadre \\
\hline Anne & $\mathrm{F}$ & 15 & $\begin{array}{l}\text { Banlieue } \\
\text { parisienne }\end{array}$ & Pavillon & $\begin{array}{l}\text { Chez ses } \\
\text { parents }\end{array}$ & Brevet & Collégienne \\
\hline Audrey & $\mathrm{F}$ & 26 & $\begin{array}{l}\text { Banlieue } \\
\text { parisienne }\end{array}$ & Pavillon & $\begin{array}{l}\text { Mariée } \\
\text { Bébé }\end{array}$ & BEP & Coiffeuse \\
\hline Chantal & $\mathrm{F}$ & 67 & Paris & Appartement & Séparée & Bac & $\begin{array}{l}\text { Sans } \\
\text { profession }\end{array}$ \\
\hline Claude & $\mathrm{M}$ & 62 & Rhône-Alpes & Maison village & Marié & $\mathrm{Bac}+2$ & $\begin{array}{l}\text { Instituteur } \\
\text { retraité }\end{array}$ \\
\hline Evelyne & $\mathrm{F}$ & 60 & Rhône-Alpes & Maison village & Mariée & $\mathrm{Bac}+2$ & $\begin{array}{l}\text { Institutrice } \\
\text { retraitée }\end{array}$ \\
\hline Hervé & M & 43 & $\begin{array}{l}\text { Banlieue } \\
\text { parisienne }\end{array}$ & Pavillon & $\begin{array}{l}\text { Marié } \\
\text { Enfants }\end{array}$ & $\mathrm{Bac}+2$ & Cadre \\
\hline Jan & M & 30 & Paris & Locataire & Célibataire & $\mathrm{Bac}+5$ & Chômeur \\
\hline Jean-Marc & $\mathrm{M}$ & 58 & Rhône-Alpes & $\begin{array}{l}\text { Appartement } \\
\text { périphérie }\end{array}$ & Marié & $\mathrm{Bac}+5$ & Professeur \\
\hline Jérémy & $\mathrm{M}$ & 19 & $\begin{array}{l}\text { Banlieue } \\
\text { parisienne }\end{array}$ & Maison de ville & $\begin{array}{l}\text { Chez ses } \\
\text { parents }\end{array}$ & BEP & Lycéen \\
\hline Laurence & $\mathrm{F}$ & 45 & $\begin{array}{l}\text { Banlieue } \\
\text { parisienne }\end{array}$ & Pavillon & $\begin{array}{l}\text { Mariée } \\
\text { Enfants }\end{array}$ & $\mathrm{Bac}+2$ & Cadre \\
\hline Lucie & $\mathrm{F}$ & 19 & $\begin{array}{l}\text { Banlieue } \\
\text { parisienne }\end{array}$ & Maison de ville & $\begin{array}{l}\text { Chez ses } \\
\text { parents }\end{array}$ & BEP & Lycéenne \\
\hline Madeleine & $\mathrm{F}$ & 85 & Rhône-Alpes & Pavillon & Veuve & Brevet & Retraitée \\
\hline Maria & $\mathrm{F}$ & 49 & $\begin{array}{l}\text { Banlieue } \\
\text { parisienne }\end{array}$ & Maison de ville & $\begin{array}{l}\text { Mariée } \\
\text { Enfants }\end{array}$ & CAP & $\begin{array}{l}\text { Femme de } \\
\text { ménage }\end{array}$ \\
\hline Marie & $\mathrm{F}$ & 41 & Paris & Studio & Célibataire & $\mathrm{Bac}+5$ & $\begin{array}{l}\text { Cadre au } \\
\text { chômage }\end{array}$ \\
\hline $\begin{array}{l}\text { Marie- } \\
\text { Claude }\end{array}$ & F & 68 & Limousin & Maison village & $\begin{array}{l}\text { Mariée } \\
\text { Grand-mère }\end{array}$ & Brevet & $\begin{array}{l}\text { Sans } \\
\text { profession }\end{array}$ \\
\hline Sylvie & $\mathrm{F}$ & 55 & Rhône-Alpes & $\begin{array}{l}\text { Appartement } \\
\text { périphérie }\end{array}$ & Mariée & $\mathrm{Bac}+2$ & $\begin{array}{l}\text { Sans } \\
\text { profession }\end{array}$ \\
\hline Thierry & $\mathrm{M}$ & 28 & Normandie & $\begin{array}{l}\text { Pavillon petite } \\
\text { ville }\end{array}$ & Célibataire & $\mathrm{Bac}+5$ & Cadre \\
\hline
\end{tabular}


ANNEXE A3 : Guide d'entretien

Début de l'entretien en mode non directif :

«Qu'est-ce qui vous fait peur par rapport à votre alimentation ? Pourquoi ? » On demandera au consommateur de présenter sa cuisine (ustensiles, aliments, ...)

\section{Exercices projectifs :}

- Soumettre une liste de risques alimentaires (voir grille) et faire réagir selon des méthodes projectives : associer ces risques à des photos de personnes fournies par nos soins (ayant l’air en bonne santé, en surpoids, malades, ... ) : voir $1^{\mathrm{er}}$ jeu de photos

- Montrer des photos de plats emblématiques et faire réagir [part de l'objectif et du subjectif] : voir $2^{\mathrm{è}}$ jeu de photos

Poursuite de l'entretien en mode semi-directif :

- S’ils n’ont pas été abordés, relancer sur les thèmes suivants :

o Quelle importance accordez-vous à votre alimentation et quel rôle joue-t-elle sur vous ? [santé, bien-être, beauté]

o Que signifie se faire plaisir quand on mange ? Quand vous voulez vous faire plaisir en mangeant, que mangez-vous? [plaisir, interdit, transgression, naturalité]

o Votre alimentation est-elle source d'angoisse pour vous ? Pourquoi ?

o L'alimentation de vos proches est-elle source d'angoisse pour vous ? Pourquoi ?

o Est-il difficile de manger sainement ? Quelles sont vos solutions ?

o Comment évaluez-vous si un produit est bon pour votre santé ?

o A qui/quoi vous fiez-vous pour obtenir des informations nutritionnelles? Vs à qui/quoi ne vous fiez-vous pas, qu'est-ce qui vous alerte? Regardez-vous les indications sur les emballages ? Que pensez-vous des allégations nutritionnelles?

o Exemples de marques, labels, entreprises qui savent vous donner confiance sur la qualité nutritionnelle de leurs produits et pourquoi. Inversement, contre-exemples?

- En fin d'entretien, on notera les informations suivantes sur le consommateur :

o prénom, âge, lieu d’habitation

o situation familiale, nombre et âge des enfants

o niveau d'étude, profession, revenus du foyer

o idée du montant des dépenses pour la nourriture par semaine ou mois du foyer

o état de santé et celui de ses proches.

- Demander la communication des tickets de caisse des courses alimentaires pendant les 15 prochains jours, laisser une pochette pour ce faire et prendre rendez-vous dans 15 jours. 


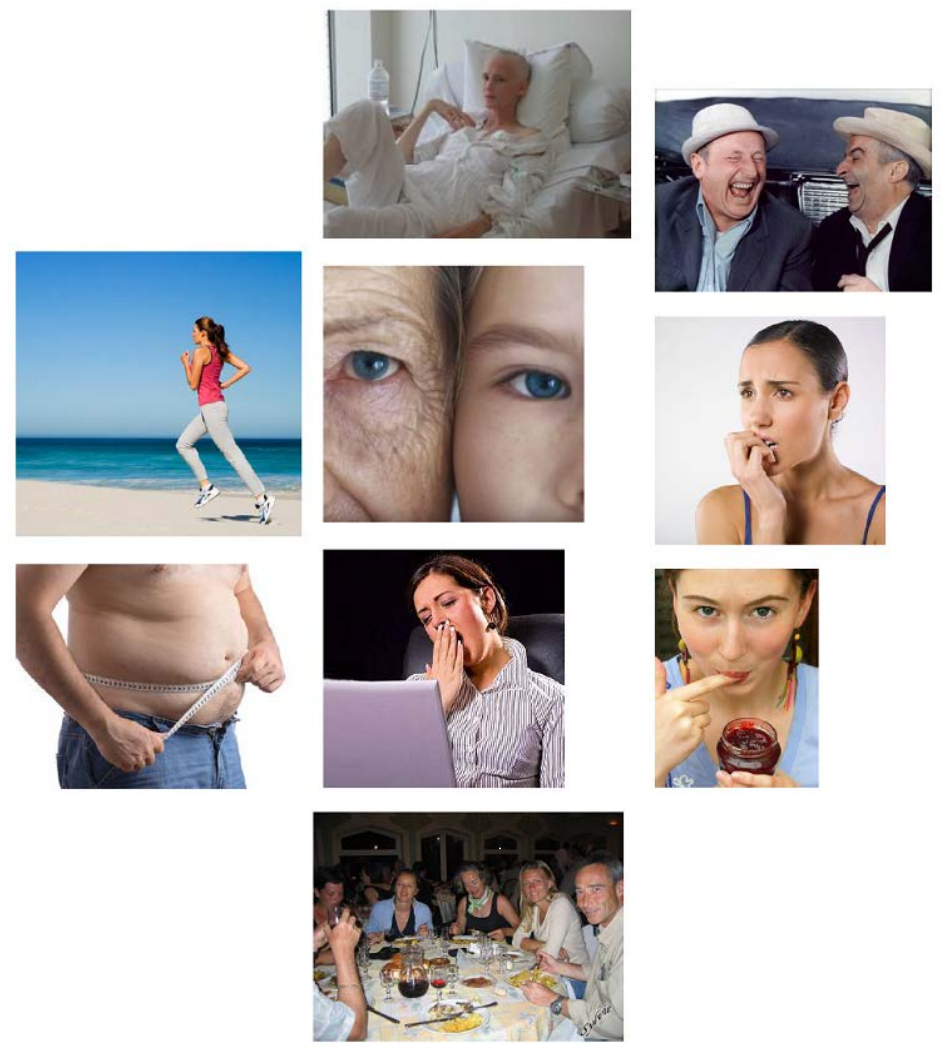

1er jeu de photographies : exercice sur les risques alimentaires
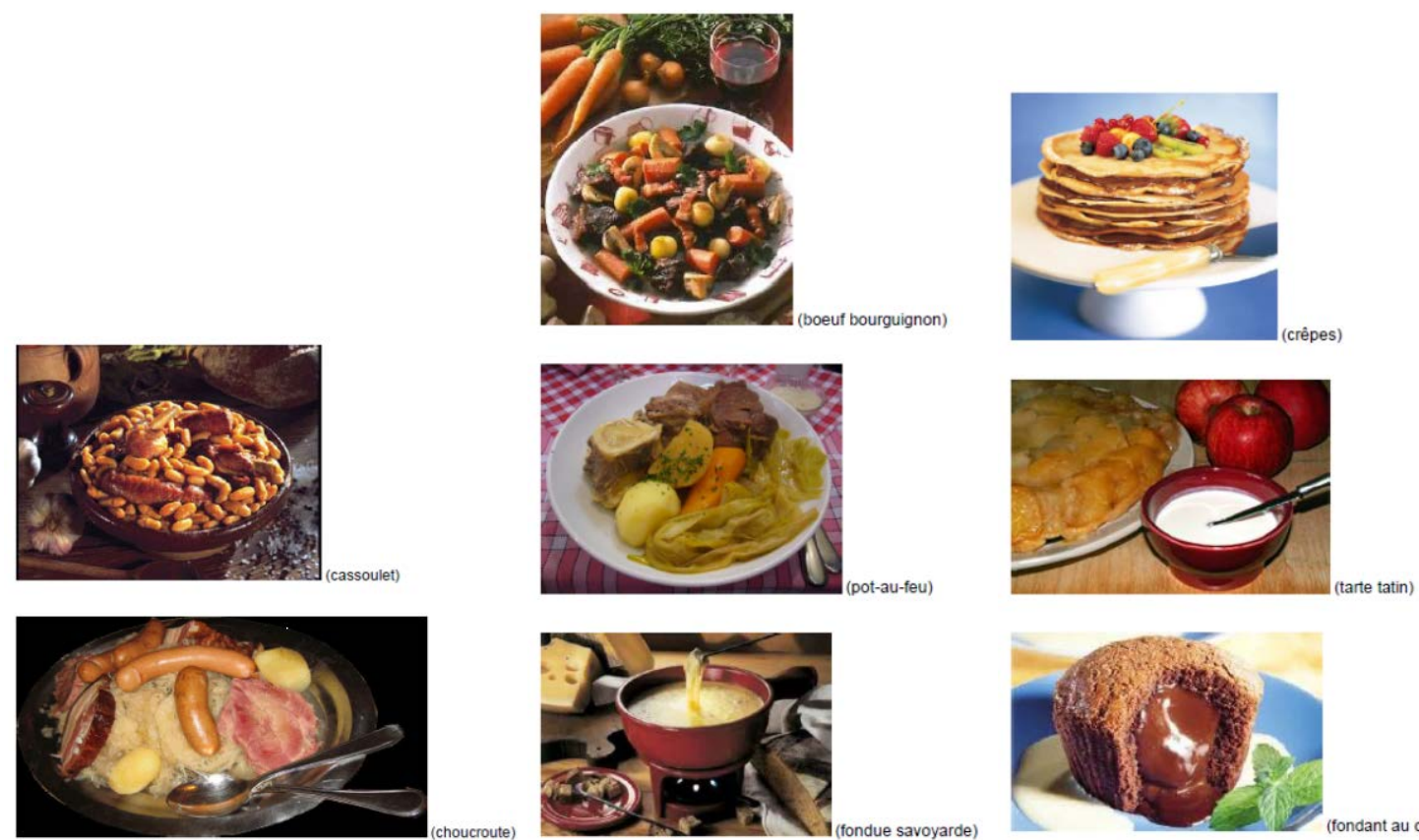

2è jeu de photographies : exercice sur les plats emblématiques 


\begin{tabular}{|c|c|c|c|c|c|c|c|c|c|c|c|c|}
\hline $\begin{array}{l}\text { Risques puis plats (chacun } \\
\text { présenté dans un ordre } \\
\text { aléatoire) }\end{array}$ & 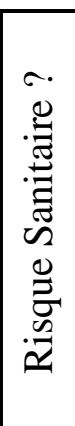 & 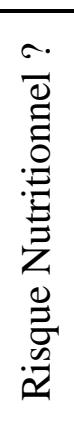 & 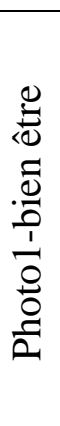 & 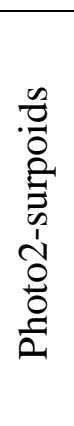 & 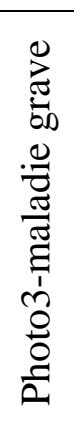 & 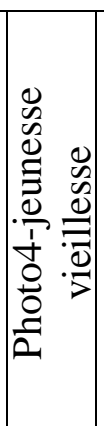 & 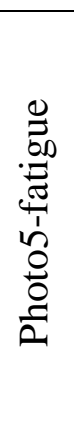 & 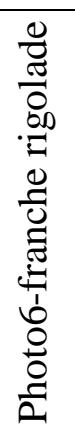 & 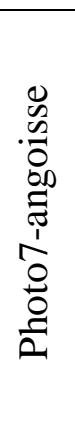 & 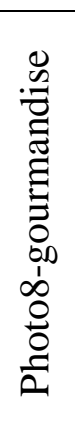 & 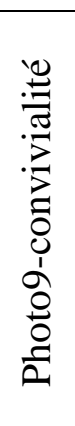 & 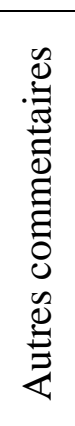 \\
\hline \multicolumn{13}{|c|}{ A) Risques alimentaires } \\
\hline Manger du fromage au lait cru & $\mathrm{X}$ & $\bar{X}$ & & & & & & & & & & \\
\hline $\begin{array}{l}\text { Aliment avec une forte teneur } \\
\text { en matière grasse }\end{array}$ & & $\bar{X}$ & & & & & & & & & & \\
\hline $\begin{array}{l}\text { Aliment avec une forte teneur } \\
\text { en sucre }\end{array}$ & & $\bar{X}$ & & & & & & & & & & \\
\hline $\begin{array}{l}\text { Aliment avec une forte teneur } \\
\text { en sel }\end{array}$ & & $\mathrm{X}$ & & & & & & & & & & \\
\hline Présence de pesticides & $\mathrm{X}$ & $\mathrm{X}$ & & & & & & & & & & \\
\hline $\begin{array}{l}\text { Longues listes d'ingrédients } \\
\text { avec additifs }\end{array}$ & & $\bar{X}$ & & & & & & & & & & \\
\hline OGM & $\mathrm{X}$ & $\mathrm{X}$ & & & & & & & & & & \\
\hline Radioactivité & $\mathrm{X}$ & & & & & & & & & & & \\
\hline Maladie de la vache folle & $\mathrm{X}$ & & & & & & & & & & & \\
\hline \multicolumn{13}{|c|}{ B) Plats emblématiques } \\
\hline \multicolumn{13}{|l|}{ Citations spontanées } \\
\hline \multicolumn{13}{|l|}{ Citations spontanées } \\
\hline \multicolumn{13}{|l|}{ Citations spontanées } \\
\hline \multicolumn{13}{|l|}{ Cassoulet } \\
\hline \multicolumn{13}{|l|}{ Choucroute } \\
\hline \multicolumn{13}{|l|}{ Bœuf bourguignon } \\
\hline \multicolumn{13}{|l|}{ Pot-au-feu } \\
\hline \multicolumn{13}{|l|}{ Raclette } \\
\hline \multicolumn{13}{|l|}{ Crêpes } \\
\hline \multicolumn{13}{|l|}{ Tarte tatin } \\
\hline Fondant au chocolat & & & & & & & & & & & & \\
\hline
\end{tabular}

Trame utilisée pour chaque répondant pendant les exercices projectifs 
ANNEXE A4 : principaux verbatim représentatifs du thème

\section{NATURALITE PERCUE}

\section{Critère des ingrédients : suspicion face aux longues listes compliquées}

"Tu peux voir si un produit est bas de gamme ou pas je pense en regardant le nombre d'additifs qu'il y a dedans. (...) Dans [un produit] de qualité, y aura presque pas d'additifs, et si y a dix additifs, c'est qu'il y en a un qui corrige l'autre, pis l'autre qui corrige l'un. C'est de la cuisine industrielle, où on compense la piètre qualité des matières premières par des exhausteurs de goût et des choses comme ça » (Jean-Marc).

"Ce que j'appelle produit suspect, c'est ce que je peux pas identifier, là en bon français. Si maintenant je vois qu'une soupe de tomate contient (...) pommes de terre, tomates, carottes, là OK, j'ai compris, sel, poivre, lait, moutarde, citron, OK. Dès que c'est dextrose, amidon modifié, déjà, là, euh, je me demande pourquoi, enfin je comprends pourquoi, c'est des épaississants, des conservateurs, et après si y a des E bidules et des A machins, je prends pas» (Marie).

"J'ai tendance à penser que pour que ce soit sain, il faut le moins d'ingrédients possible. (...) Quand il commence à y avoir une liste un peu longue, y a une idée un peu plus chimique derrière, de traitement, de, d'élaboré quoi. (...) Dans ma tête, c'est un traitement plus industriel, et moins axé sur le goût et plus axé sur de la production en quantité, en rendement. (...) L'artisanal, c'est un gage de meilleur goût, oui, c'est l'image que j'ai, c'est que c'est plus axé sur les qualités nutritionnelles du produit, (...) par rapport à un truc industriel qui va plutôt être axé, dans mon esprit, sur la rentabilité économique de la production. (...) [Je préfère prendre] le truc le plus pur possible et faire le mélange moi derrière quoi » (Thierry).

\section{Critère de la transformation : plébiscite de la cuisine maison}

"Moi je suis plutôt axée sur les produits simples, pas transformés. Quand je regarde la composition des produits transformés, je trouve toujours des rajouts de produits qui me semblent pas du tout indispensables pour, euh, pour cuisiner quoi. (...) Si je pars de produits simples, je me dis que je mange plus sainement que si je prends des produits manufacturés, transformés » (Sylvie).

«Tout ce qui est plats cuisinés, j’achète pas. Je sais pas ce qu'il y a dedans. Je fais pas confiance, non. (...) Je préfère le faire moi-même » (Maggy).

" Je me demande toujours ce qu'il y a dans les plats cuisinés, je préfère faire moi-même, c'est meilleur. Je me dis toujours, qu'est-ce qu'ils ont été mettre dedans de chimique, je suis un peu psychopathe avec ça » (Audrey).

"Les produits transformés, d'un point de vue nutritionnel, ben y a plus rien dedans. (...) T'es trompée en fait dans ton organisme, t'es trompée sur la marchandise [avec les] produits industriels » (Laurence).

«Du lait qui est supplémenté en vitamines, je prends pas. Je me dis si j'ai besoin de vitamines, elles sont forcément apportées par l'aliment qui doit me l'apporter. Si y en a pas dans le lait, c'est que ça doit pas se trouver dans le lait. Si y a un jus de fruit enrichi en fer, je prends pas. Je préfère un jus de fruit normal, sans rien d'ajouté » (Marie).

«Je pense que les plats préparés que j’achèterais, type lasagnes, ou hachis Parmentier, sont moins bons que ceux qu'on fait nous-mêmes, au goût et pour la santé : (...) je suis persuadée que y a plein de conservateurs et de choses comme ça dedans » (Adeline).

« J'aime pas trop les trucs tout prêts. (...) Au début, je ne cuisinais quasiment pas, ou des choses toutes prêtes, mais là de plus en plus, j'essaie soit de cuisiner moi-même, soit de manger quelque chose qui est cuisiné par mes parents » (Jan).

«Un gâteau fait-maison, oui. (...) C'est pas un gâteau industriel, enfin ça sort pas d'une usine, (...) c'est mieux quoi. Même pour la santé. Alors que, un gâteau industriel, on sait pas ce qu'ils mettent dedans. (...) Tout ce qui est industriel, c'est jamais bon pour la santé, hein » (Lucie). 


\section{Critère de l'apparence : rejet du standardisé trop éloigné du naturel}

«Même les fruits et légumes, ils ont tous la même forme, c'est parce qu'on (...) les force à pousser avec des produits, je crois que c'est les produits phytosanitaires.(...) Les vrais fruits, les citrons, ils sont sales. (...) Les citrons, là ils se ressemblent tous, ils ont tous du produit chimique à l'intérieur » (Lucie).

«Ca me dérange pas d'avoir une carotte moche par exemple. (...) Parce que je trouve logique que la nature fasse (...) des carottes créatives. Des trucs calibrés, ça moi je... Je trouve dommage qu'on jette des petites carottes parce qu'elles sont pas du calibre que le supermarché a retenu. Ca moi je trouve ça... inadmissible quoi. Donc ce qu'il y a de la nature, du végétal, ça pour moi c'est bon » (Marie).

«Un beau produit bien brillant, bien calibré et tout, pour moi ça signifie pas forcément la qualité, loin de là. Ça signifie plus la standardisation. Donc pas forcément un produit fait pour être bon, mais un produit fait pour être beau. A la limite, c'est suspect » (Claude).

\section{Critère du temps et du lieu de production : respect des saisons, préférence locale}

« J'essaie quand même de prendre des produits de saison, et du coin » (Laurence).

«Je regarde la provenance. Parce qu'elle me donne une indication sur la saison. Si la fraise vient du Chili, j'achète pas. Je préfère pas manger » (Marie).

«Il est très important de suivre les saisons. Il faut respecter le rythme naturel » (Claude).

«A chaque saison, j'ai le produit. J'ai toujours des produits très frais. Je ne mange pas de produits hors saison. (...) Une alimentation saine, c'est justement manger les légumes de saison. Ça c'est très important. C'est-à-dire pas manger des tomates en hiver, des choses comme ça » (Marie-Claude).

\section{Critère du lieu d'achat : soupçons face à la grande distribution et à la restauration collective}

" J'ai souvent entendu dire, c'est meilleur d'acheter chez le petit commerçant, afin de faire son petit truc à soi, à la maison. (...) Je préfère le fait-maison, je sais ce qu'il y a dedans, c'est moi qui le mets, je sais où je vais le chercher » (Audrey).

«Tu prends l'exemple du boucher, on sait qu'on peut avoir confiance en ses produits (...), sur la façon dont déjà il choisit la matière première et sur la façon dont il la transforme. (...) Quand on voit derrière, au supermarché, les bouchers préparer leur viande, bon ils sont là, ils font leurs huit heures, ils font ça d'une manière, euh, industrielle disons. Tandis que lui il fait ça avec... on le voit travailler avec ses apprentis, il fait ça avec amour, l'amour du métier bien fait » (Claude).

"Quand je vais chez mon boucher, t'as le côté gourmandise aussi, j'ai envie de tout acheter chez lui. Et en plus, je sais que c'est des bonnes vaches (...).Alors que moi, quand je vois la viande en supermarché, sous vide et... ou même, ils ont des bouchers, je trouve ça, je peux rester à marcher dans le rayon de viande pendant trois - quatre minutes, à rien prendre » (Laurence).

«En grande surface, ça me fait peur, je me dis est-ce que ça va être frais, est-ce que ça va être bon, (...) surtout par rapport à la viande. (...) Pour les fruits, sur le marché, y a un petit marchand, que j'aime bien, (...) et j'ai le petit épicier arabe (...). Je connais, donc c'est plus rassurant. (...) En grande surface, j'aime pas trop, les fruits, ils mettent toujours un tas de truc dessus » (Audrey).

«Le fondant au chocolat, pour moi c'est l'exemple type en restaurant du produit industriel. Ça signifie que c'est pas bon. C'est vraiment pas bon ni au goût ni pour le corps.(...) J'étais assez naïve pour penser que tous les produits qu'étaient en restaurant étaient faits dans le restaurant » (Marie).

«Ce qui me fait peur, c'est la valeur nutritionnelle des ingrédients qu'on mange, surtout quand on ne mange pas chez soi, (...) quand tu manges au restaurant » (Laurence)». 
ANNEXE A5 : principaux verbatim représentatifs du thème

\section{COMMENSALITE}

\section{Le rôle rassurant du terroir, emblématique du partage}

«La choucroute, ça c'est aussi un plat du partage, de l'hiver, un truc exceptionnel » (Marie).

"La raclette, c'est le truc que typiquement, je mangerais jamais tout seul, (...) qui se mange un soir où t'as pris l'air dans la journée et t'es avec des gens que t'aimes bien et t'as un truc convivial à la bonne franquette » (Thierry).

«Pour moi, le pot-au-feu, c'est un plat d'hiver, ça réchauffe. (...) Il fait mauvais dehors, tu manges un bon potau-feu. C'est rassurant » (Claude).

«La tarte tatin, c'est le plaisir entre amis ou en famille » (Adeline).

"Ah la raclette c'est délicieux, hein. Ca évoque la famille. En général c'est quand mes petits-enfants viennent » (Madeleine).

«Pour moi c'est des plats qui se mangent nombreux, ça. C'est les différentes générations, les plats de grandsmères pour les enfants aussi » (Adeline).

«La fondue savoyarde, pour moi, c'est un peu une histoire de réunion de famille, le plat du terroir » (Thierry).

"L'idée d'un repas de famille. C'est le genre de repas finalement un peu de transmission, de passation» (Evelyne).

« Pourquoi pas, entre potes, se faire un cassoulet, voilà » (Laurence).

«Les produits du terroir, c'est quelque chose qu'on partage, oui. Qu'on partage et qu'on a envie de faire partager » (Hervé).

\section{Au-delà, le plaisir à partager son alimentation}

«Ce qui prime, c'est d'être ensemble, et de rigoler, et de partager, de parler de soi, et la nourriture est un prétexte pour rester un moment plus long ensemble » (Marie).

"C'est pas tant lié à ce qu'on mange qu'à la, la circonstance quoi. Par exemple, quand j'ai des copains qui viennent à la maison, finalement j'ai tendance à leur faire à manger quelque chose qu'est pas très différent de ce que je mange moi, mais l'ambiance est très différente parce que c'est une convivialité autour » (Thierry).

"C'est la signature de la France, bien manger, ouais. Manger, partager. Je pense que ça va ensemble, même. Si tu manges tout seul, c'est pas drôle » (Hervé).

«T'as la nourriture-plaisir, partager un repas avec des amis, de la famille » (Sylvie).

«Je me dis que le plaisir, je devrais le prendre lors des repas entre amis. En effet, pouvoir aller chez une amie qui a préparé une bonne tarte tatin, là je devrais me dire, tu en prends, tu te fais plaisir, en plus tu fais plaisir à la personne qui te reçoit, tu partages avec les autre. Mais ça doit pas devenir le quotidien » (Marie).

«Manger, c'est un plaisir partagé, et ça, y a rien de mieux quoi. Parce que c'est un plaisir, c'est un petit moment de plaisir ! »(Chantal).

"C'est sûr que la cantine c'est pas bon (...). Par contre, ça te met dans l'ambiance d'une équipe, de, d'un partage. (...) La cantine c'est aussi une façon de s'ouvrir au social » (Marie).

"Recevoir c'est aussi le plaisir de te lancer dans de la cuisine que tu ferais pas pour toi tout seul tous les jours. Par exemple, moi j'aime bien faire des gâteaux, ben je ne me fais pas un gâteau pour moi. Ca me fait pas plaisir en fait. Moi ce que j'aime bien, c'est préparer un gâteau pour les gens qui vont venir. Voire de l'amener au boulot une fois de temps en temps » (Thierry). 


\section{L'aspect déculpabilisant de la commensalité}

«Le Nutella c'est une supercherie, c'est incroyable c'est super bon, mais c'est dégueulasse ce produit. (...) Lorsque tu regardes la liste des ingrédients, y a même pas de chocolat dans ce truc-là. (...) Mais tu pars en week-end avec les copains, et y a du Nutella pour les enfants, et tu te fais une bonne tartine, tu trouveras le Nutella exceptionnel, et t'auras raison d'en manger. (...) Toutes ces gourmandises sont d'autant plus gourmandes et appréciables lorsqu'elles sont exceptionnelles et partagées. Lorsqu'elles sont vécues en solitaire, ou sur des produits de mauvaise qualité, (...) là ce n'est plus un plaisir, non : après, tu culpabilises » (Marie).

"Quand tu partages, tu peux te dire que tu manges beaucoup, ça, ça te stresse pas. Alors que je pense si tu es tout seul et que tu manges beaucoup, là ça te stresse. Quand tu partages, tu t'en aperçois pas. Même si tu manges la même quantité. (...) A plusieurs, manger beaucoup, c'est, tu te sens moins euh... Bien manger c'est partager » (Hervé).

"Les repas familiaux ou avec des copains, c'est pas une source de stress. Alors que quand je suis toute seule, pff, tout le temps » (Lucie).

"Quand on est entre amis ou en famille, (...) c'est normal de se faire plaisir. [La culpabilité], ça arrive plutôt (...) en rentrant un soir de la semaine, où voilà j'ai particulièrement faim, je me jette sur la nourriture, puis après je vais me dire, oh là là, j'ai trop mangé, c'était idiot » (Adeline).

"Moi j'ai pas envie de manger tout seul. Donc euh, assez automatiquement, c'est quand même devant la télé en regardant quelque chose. Ce qui change l'état d'esprit dans lequel tu manges quoi. Pis t'as un peu tendance à manger machinalement parce que tu t'intéresses plus à ce qui se passe à la télé qu'à ce que tu manges » (Thierry). 
ANNEXE A6 : principaux verbatim représentatifs du thème

\section{HYPERCHOIX}

\section{Le choix, nécessaire pour assurer la variété nutritionnelle}

«Je pense qu'il est bon pour la santé de manger équilibré et varié. (...) Il faut que je propose un maximum d'aliments pour que ça amène au corps un maximum de choses quoi » (Adeline).

" [Ma fille] mange très mal. (...) Pourtant je me bats. (...) Mais plus ça va, plus elle mange mal d'un point de vue nutritionnel. (...) Elle va manger seulement, ouais, ce qu'elle aime. En petite quantité. Mais ce qu'elle aime, c'est quand même, d'un point de vue nutritionnel, c'est nul quoi : (...) Nutella, pizza » (Laurence).

«McDo, globalement c'est de la malbouffe parce que c'est toujours la même chose, (...) pas variée, pas équilibrée » (Adeline).

"Avec une alimentation variée, en gros, tu apportes au corps tout ce qu'il faut, au lieu de prendre des compléments. Normalement, si tu manges suffisamment de fruits, de légumes, mais aussi de sucres lents, t'arrives à avoir les apports qu'il faut. (...) Si tu n'as pas de soucis de santé, (...) tu devrais pas avoir besoin de prendre des suppléments » (Evelyne).

"Si tu manges équilibré, varié, etc., t'as pas besoin de prendre d'alicaments. C'est si tu as une carence en quelque chose » (Adeline).

\section{Le choix anxiogène}

"C'est plus un truc relatif qu'absolu quoi. C'est de dire, ben tiens, la sauce tomate, y a trois marques différentes dans le rayon, et de regarder entre les trois » (Thierry).

«Le nutritionniste m'a dit, (...) regardez ce qu'il y a dans les aliments. Et c'est pour ça qu'il m'a incitée à aller dans les magasins bio, ce que j'ai fait, et même en magasin bio, regarder, car c'est pas le bio qu'il m'a conseillé, c'était le contenu » (Marie).

"Je regarde le choix, et si y a des choses assez semblables, et y en a un dans lequel y a marqué bio ou je vois que ça a l'air moins, euh, moins sujet à ce genre de menaces, je vais prendre celui-là » (Adeline).

"Notre peur, c'est de choisir (...) la bonne alimentation. C'est, devant l'opulence, être sûr d'avoir fait le bon choix. (...) Alors qu'avant, ils pouvaient pas avoir peur de l'alimentation, puisque, y avait pas le choix en fait, c'est le choix qui fait, qui pourrait donner la peur. (...) Avant, y avait pas à gérer le trop, puisque, si on leur donnait, euh, des blettes, ils se posaient pas la question de savoir comment elles avaient poussé. (...) Nous, notre problème, c'est pas de manger, parce qu'on aura toujours à manger, c'est plutôt de choisir, c'est se dire que j'espère que j'ai mangé la bonne chose (...). C'est d'avoir le choix en fait » (Hervé)

«Nous [pendant la seconde guerre mondiale] on a tellement été privés qu'on est moins regardants peut-être que la nouvelle génération qui ont toujours eu tout ce qu'ils voulaient. Parce que nous, comme on a été privés, je pense qu'on est moins... on regarde mieux la satisfaction que ça nous apporte de manger plutôt que d'être trop, comment dire, pas délicats, mais... difficiles » (Madeleine).

\section{Limiter le choix alimentaire chez soi}

«Dans notre frigo, on a rarement des choses très riches, en calories » (Hervé).

« J'ai juste une boîte de biscuits que je n'aime pas, pour si quelqu'un vient à l'improviste. (...) Je les mangerais si j'aimais. (...) Je suis obligée de me mettre des barrières, de pas avoir de Nutella et tout ça » (Marie).

"Les menus roulants d'Adeline, c'est très bien. On mange plus varié qu'avant. Elle les fait par cinq semaines, donc pendant cinq semaines, c'est des choses différentes, puis on recommence » (mari d'Adeline).

«J'essaie de varier, viande blanche, viande rouge ; poisson euh c'est plus difficile, je le réserve plutôt pour le week-end » (Adeline). 


\section{Repérer des indicateurs de qualité extrinsèques : les premiers prix synonymes de sous- qualité nutritionnelle}

«On ne prend jamais le prix vraiment discount, on évite. Enfin, à moins qu'on ait bien regardé la composition, mais c'est quelque chose qu'on évite » (Evelyne).

«Les marques de prix le moins cher, c'est (...) au détriment de la qualité. (...) C'est l'expérience de [quand j'étais étudiant], où on avait découvert des trucs, comme le fromage râpé qu'a pas le droit de s'appeler fromage râpé, c'est de la spécialité fromagère râpée, parce que ça a même pas le droit de s'appeler fromage (rire). (...) Ou le saucisson pouce qu'avait marqué les esprits parce que la seule façon d'en manger, c'est de te rappeler le prix que tu l'as payé parce que sinon tu peux pas quoi (rire)! Donc ça clairement, c'est de, c'est du repoussoir, [pour] la qualité gustative [comme pour] l'aspect nutritionnel. (...) Par exemple, le saucisson pouce, clairement, y a moins de viande et plus de gras que le saucisson d'une autre marque » (Thierry).

« Je veux pas passer trop de temps, je fais en une demi-heure mes courses sur Télémarket. (...) Mes critères, c'est de pas acheter de bas de gamme, parce que j'ai l'impression que c'est moins bon. (...) Je me mets toujours en milieu de gamme en fait. (...) Si c'est premier prix, c'est qu'ils ont vraiment essayé de baisser tous les prix, donc ils ont forcément (...) rogné sur certains critères et donc ça doit être moins bon pour la santé. (...) Ca me sert à situer les produits, c'est une échelle » (Adeline).

«Je me dis : sous-prix, sous-produit, donc y a quelque chose en moins que le produit de base d'une marque que je connais » (Marie).

\section{Repérer des indicateurs de qualité extrinsèques : marques et labels}

«Pour certains produits, je vais forcément acheter des marques en fait » (Laurence).

« La marque, ça me rassure, si c'est connu oui ça me rassure » (Maggy).

« Je vais me mettre en milieu de gamme, et après je vais regarder les différentes marques, mais y a des marques qui me, que j'utilise plus, par habitude et que j'aime bien. Donc de toute façon je vais avoir tendance, s'ils ont un produit dans cette marque-là, d'aller sur cette marque-là. (...) Pour moi la marque, c'est plus un repère, j'ai déjà acheté une marque, et donc du coup, ça m'a plu, et donc je vais continuer à acheter soit le même produit, soit un autre produit dans la même marque » (Adeline).

«Si je vois Danone, je me dis que c'est une boîte sérieuse. Mais c'est un peu comme AB. C'est-à-dire que j'aurai pas de mauvaise surprise avec Danone (...) Le yaourt nature que j'ai habituellement au frigo, c'est un yaourt Danone. C'est le seul que j'achète. Je me dis, ça c'est un produit de base, je fais confiance au yaourt nature Danone. Je dirais que ça fait partie de mon patrimoine. Je n'imagine pas euh... ne pas faire confiance à cette marque qui m'accompagne depuis toujours » (Marie).

«Ça ne m'influence pas forcément, la marque. Non, le label prévaut sur la marque pour moi. Parce qu'un label bio est censé t'offrir un produit de qualité équivalente quelle que soit la marque » (Claude).

«Quelque chose m'énerve, c'est le bio à tout cran, (...) à toutes les sauces (...) pour faire vendre. (...) Il faut payer pour avoir la norme bio. T'as pas d'obligation de résultats. (...) Ca veut pas dire que le bio est mauvais, (...) mais il est pas forcément signe de grande qualité. (...) Je le prends plus comme un indicateur et comme source d'information » (Sylvie).

«Y a le poulet fermier, y a élevé en plein air, y a nourri au grain, etc. etc. c'est un peu compliqué» (Claude).

« Je vais prendre l'exemple du vin, l'Appellation d'Origine Contrôlée, c'est en rien une garantie de qualité, ah non. Donc je ne crois pas du tout aux labels. Non, tu peux prendre, je sais pas, prenons un Chambolle Musigny, donc on en a du 2008, AOC, tu en prends 10, sur les 10, y en aura 2 à boire et 8 mauvaises, toutes AOC. (...) C'est pas une garantie, c'est un filtre supplémentaire » (Jean-Marc). 
ANNEXE A7 : principaux verbatim représentatifs du thème

\section{PRN, MARQUES ET ALLEGATIONS NUTRITIONNELLES}

\section{Les allégations nutritionnelles, une information à prendre avec du recul}

"[Les allégations nutritionnelles], c'est quelque chose que je regarde, mais je sais pas si j’y suis très sensible. Enfin, ça dépend des informations qui sont données. (...) C’est difficile de savoir » (Sylvie).

«Je trouve que c'est assez peu lisible. Déjà il faut presque une loupe! Et puis bon, c'est vrai que je fais pas vraiment attention à ça. (...) Je ne dis pas que je n'y crois absolument pas, mais c'est à modérer. Il y a l'exagération, il faut moduler. Mais les allégations ne sont pas non plus forcément fausses et inutiles » (Evelyne).

«Si y a marqué en effet sans ajout, là je vais vérifier, mais ça va attirer mon attention. (...) Bon, ce qui me fait marrer, c'est quand on dit que l'eau d'Evian est sans sucre ajouté. Parfois ils se moquent un peu du monde. Y a eu des trucs comme ça. Ou c'était la Volvic aromatisée, sans sucre ajouté, ben j'espère bien. Ou trente fois plus de sucre en moins, des trucs comme ça, ça m'agace. Mais euh... Je vérifie » (Marie).

«Oui, bon alors, il y a tout ce qui se dit et qui n'a aucune valeur : produit extra, super, bon pour tout, sans aucune preuve à l'appui, alors là bon ben là (...) c'est de la tromperie, c'est de la publicité. Mais on regarde ce qui a quand même une certaine valeur établie. Faut que ce soit pas uniquement de la publicité, faut que ce soit des allégations confirmées, quoi ! » (Claude)

\section{Les marques jugées incompétentes en nutrition ${ }^{5}$}

"J'y crois pas, (...) absolument pas. Parce que je pense que c'est essentiellement de la publicité, mais qu'y a pas d'études sérieuses qui sont faites. Pour avoir des études sérieuses faites sur le lien alimentation et santé, faut un recul de trente ou quarante ans, minimum. On est loin de les avoir » (Jean-Marc).

"Actimel, moi j'y crois pas à tout ça. Et pourtant, j'ai une belle-fille agro qui travaille chez Danone. Donc pour nous, Danone, tout est bon, mais je ne crois pas à ça » (Marie-Claude).

"Je pense que c'est du pipeau. Ben parce qu'en fait, pour moi, ce qu'ils mettent sur l'emballage, c'est ce qui va faire que le client va choisir ce produit-là. Donc c'est d'abord mettre un message qui fasse que le produit il va être choisi, et ensuite, ils essaient de...Il faut que ça soit fondé sur un peu de véracité, mais... Y a rien de prouvé, et la façon dont c'est libellé est toujours (...) un peu ambiguë » (Adeline).

"Les choses $0 \%$, les choses comme ça, (...) j'y prête pas forcément attention puisque j'y crois pas. Y a pas besoin de manger des trucs enrichis en vitamines. Autant manger un bon steak, là y a plein de vitamines!» (Audrey).

«Danone, (...) je sais que c'est un message, euh, marketing, et puis, enfin pour moi, ils ont un peu surfé sur cette vague-là, et c'est pas une garantie de, de, enfin ils ont rien prouvé quoi, pour moi, là-dessus » (Adeline).

«Pub pub pub! C'est de la pub, c'est pour faire vendre. Pour moi, ça n'a pas d'effet sur la santé. (...) Il faudrait que tu manges vingt yaourts par jour pendant des années, donc tout ça c'est pour faire vendre, c'est du marketing » (Chantal).

\section{L'accusation de manque d'intégrité des marques}

«Le dicours d'Actimel, j'y crois pas. J'y crois pas du tout. (...) Je me dis, c'est du business, du marketing quoi. De toute façon, ils ont aucun intérêt à bien nous nourrir, ces gens-là. Ils sont dans le business, (...) ils ont l'image qu'ils veulent donner, mais ils sont pas là pour nous faire du bien, (...) ils sont là pour développer leurs

${ }^{5}$ La compétence et l'intégrité sont les deux dimensions de la crédibilité (Simon, Berkowitz et Moyer, 1970) 
ventes, quoi » (Laurence).

«A la fin tu te poses des questions quoi. (...) Il n'y a pas de limites. Ils peuvent être malhonnêtes aussi dans les ingrédients, ils peuvent mettre des produits de mauvaise qualité pour tirer profit de l'affaire » (Claude).

«Par exemple, fort en oméga trois, tout ça, non, je regarde pas. Parce que je n’y crois pas, non, du tout. (...) Sur les packagings des produits alimentaires, (...) ils en profitent, les producteurs, pour pas mettre non plus tous les ingrédients qui sont mis dedans en fait » (Jérémy).

"Je préfère même plus regarder les ingrédients, parce que de toute façon, tous les aliments sont, euh, pas sains » (Lucie).

«Je me dis Danone peut pas (...) commettre d'erreur dans ce qu'ils mettent dans leurs produits, parce qu'ils sont tellement surveillés, c'est un groupe énorme, y a trop d'enjeu. Mais je suis pas dupe quoi »(Marie)

"Tous les ingrédients qui sont marqués au dos des produits, c'est ce qu'ils veulent bien nous montrer. Et donc la plupart du temps donc, quand ils mettent $0 \%$ de matière grasse, c'est de la publicité mensongère, parce que bon, c'est remplacé par des, par de la matière grasse de synthèse ou des choses comme ça. Et c'est pas forcément bon pour la santé non plus » (Jérémy).

« J'ai conscience du monde business, du marketing qui nous entoure. J'essaie de pas me faire avoir. (...) Enfin côté nutrition, je sais, je veux et j'y arrive pas : c'est très frustrant quoi » (Marie). 
TABLEAUX [à intégrer dans le texte]

\begin{tabular}{|c|c|}
\hline \multicolumn{2}{|c|}{ Risque alimentaire } \\
\hline Risque sanitaire & Risque nutritionnel \\
\hline $\begin{array}{ll}\text { - } & \text { Extrinsèque à l'aliment : empoisonnement par } \\
\text { - } & \text { contamination (Ferreira, 2006) } \\
\text { - } & \text { Sortée court terme (Aurier et Sirieix, 2009) } \\
& \text { (Aurier et Sirieix, 2009) } \\
\text { - } & \text { La néophobie en protège (Fischler, 1990) } \\
\text { - } & \text { Responsabilité de la collectivité (Fischler, } \\
& 1990 \text {; Lipovetsky, 2006) }\end{array}$ & $\begin{array}{ll}\text { - } & \text { Intrinsèque à l'aliment : effets secondaires de } \\
\text { - } & \text { déséquilibres nutritionnels (Ferreira, 2006) } \\
\text { - } & \text { Portée long terme (Aurier et Sirieix, 2009) } \\
& \text { (Aurier et Sirieix, 2009 ; OMS, 1946) } \\
\text { - } & \text { La néophilie en protège (Fischler, 1990) } \\
\text { - } & \text { Responsabilité de l'individu (Fischler, 1990 ; } \\
& \text { Lipovetsky, 2006) }\end{array}$ \\
\hline
\end{tabular}

Tableau 1. Les dimensions sanitaire et nutritionnelle du risque alimentaire

\begin{tabular}{|lcc|}
\hline $\begin{array}{c}\text { Termes utilisés } \\
\text { (ou de la même famille) }\end{array}$ & Occurrences & Nombre de répondants concernés \\
\hline Cuisiner & 125 & 18 \\
Ingrédients & 69 & 16 \\
Nature & 46 & 12 \\
Simple & 28 & 12 \\
Industrie & 24 & 10 \\
Transformé & 19 & 7 \\
Standardisé / calibré & 14 & 4 \\
Traditionnel & 14 & 6 \\
Chimie & 11 & 7 \\
\hline
\end{tabular}

Tableau 2. Analyse lexicale, occurrences pour le thème de la naturalité

\begin{tabular}{|lcc|}
\hline \multicolumn{1}{|c}{$\begin{array}{c}\text { Termes utilisés } \\
\text { (ou de la même famille) }\end{array}$} & Occurrences & Nombre de répondants concernés \\
\hline Plaisir & 118 & 18 \\
Repas & 113 & 17 \\
Amis / Copains & 74 & 13 \\
Table & 41 & 15 \\
Tout seul / toute seule & 34 & 11 \\
Partage & 28 & 7 \\
Famille & 25 & 10 \\
Ensemble & 21 & 10 \\
Convivial & 18 & 8 \\
Grands-parents & 16 & 9 \\
Génération & 4 & 4 \\
\hline
\end{tabular}

Tableau 3. Analyse lexicale, occurrences pour le thème de la commensalité 


\begin{tabular}{|lcc|}
\hline \multicolumn{1}{|c}{$\begin{array}{c}\text { Termes utilisés } \\
\text { (ou de la même famille) }\end{array}$} & Occurrences & Nombre de répondants concernés \\
\hline Regarder / vérifier & 258 & 18 \\
Choix / choisir & 82 & 16 \\
Variété & 31 & 11 \\
Chercher & 31 & 15 \\
Trop de & 22 & 11 \\
Critère & 20 & 9 \\
Evaluer & 14 & 12 \\
Rationner / limiter / réduire & 12 & 7 \\
Comparer & 8 & 5 \\
Relatif & 8 & 5 \\
Décision & 8 & 6 \\
\hline
\end{tabular}

Tableau 4. Analyse lexicale, occurrences pour le thème de l'hyperchoix 
FIGURES [à intégrer dans le texte]

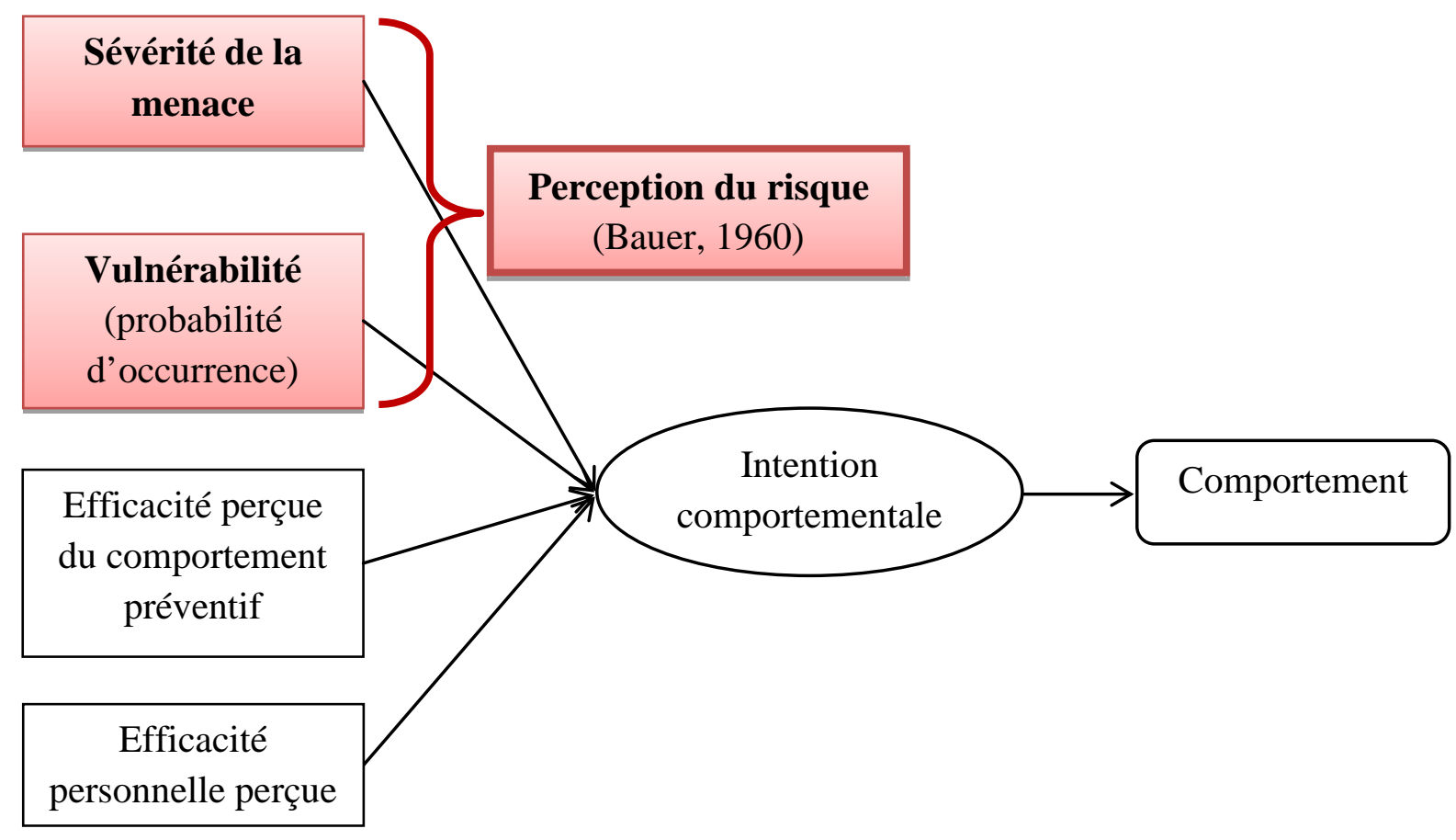

Figure 1. Place de la perception du risque dans la théorie de la motivation à se protéger de Rogers (1975 ; Maddux et Rogers, 1983)

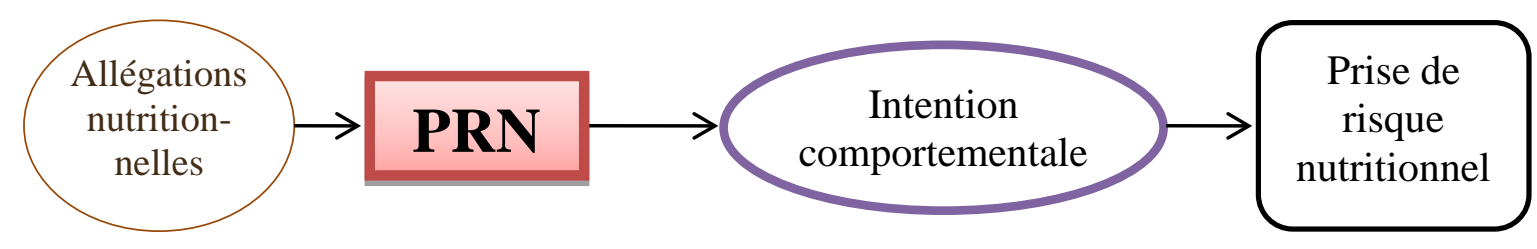

Figure 2. Mécanismes et influence de la PRN d'après la revue de littérature 
Prise de risque nutritionnel élevée

Grignotage

contre le stress

d'un effort ou en échange
Repas au

restaurant en

tête à tête

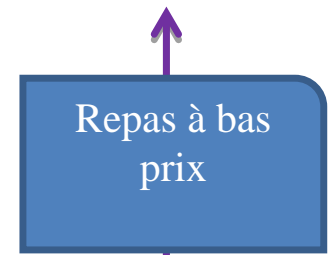

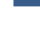
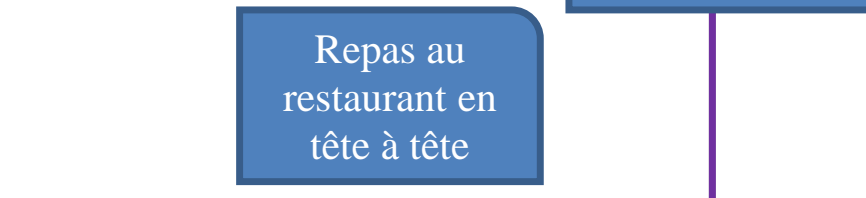

Repas dans le

cadre d'un

régime

équilibré

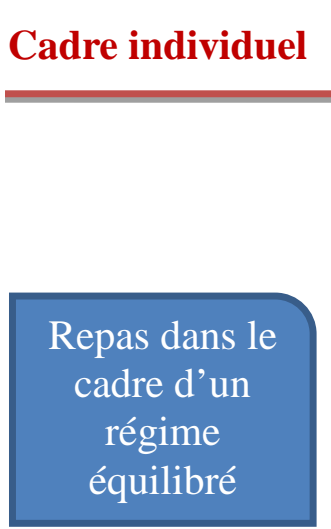

\section{Repas}

quotidiens en

famille
Fêtes

Dîners d'affaires

ou cocktails

Prise de risque nutritionnel faible

Plateaux repas

Cantine

\section{Cadre social}

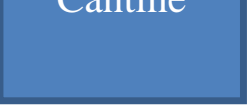

Figure 3. Une prise de risque nutritionnel différente selon le contexte de consommation

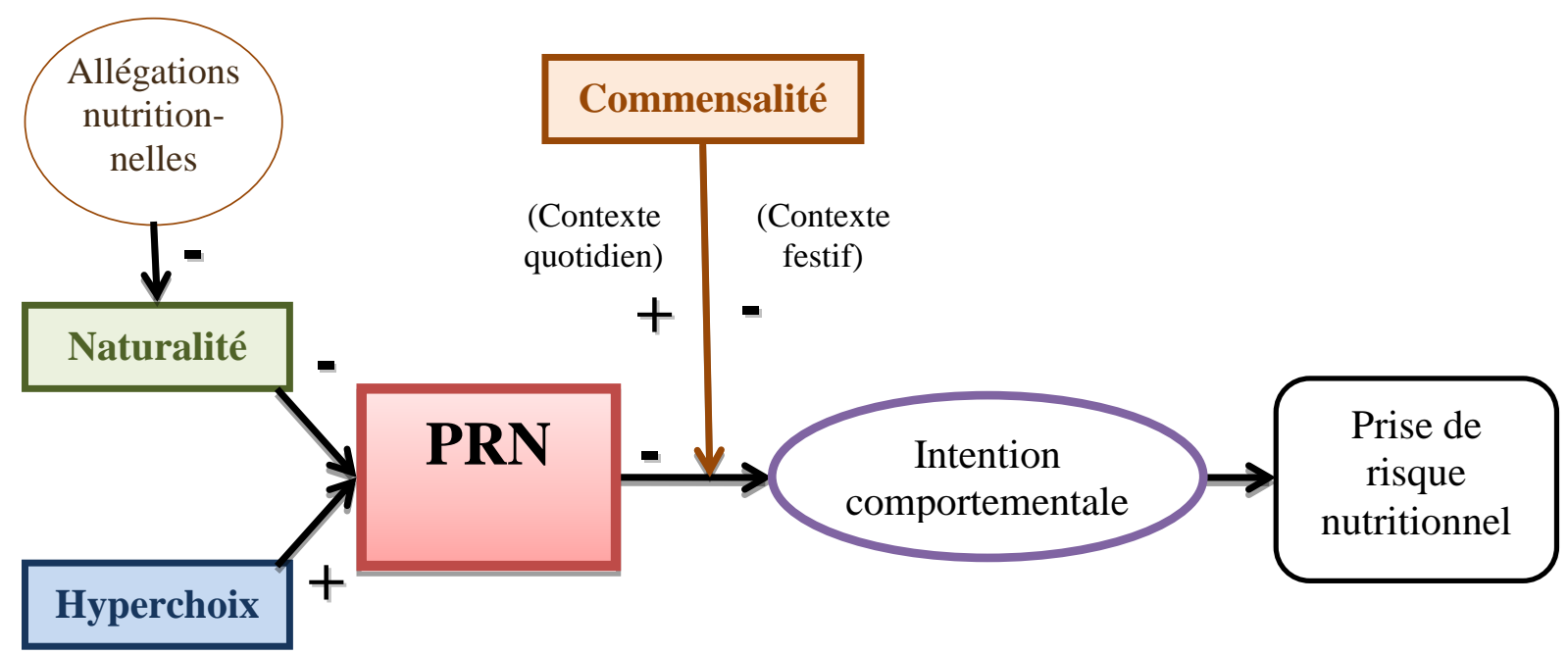

Figure 4. Proposition d'un cadre conceptuel de la PRN 


\section{REFERENCES BIBLIOGRAPHIQUES}

Afsaa et Inpees (2004), Comparaison de deux enquêtes nationales de consommation alimentaire auprès des adolescents et des adultes (Baromètre Santé Nutrition 2002 et INCA 1998-99) : éléments de méthode et résultats,

Ajzen I. (1991), The theory of planned behavior, Organizational Behavior \& Human Decision Processes, 50, 2, 179-211.

Ajzen I. et Fishbein M. (1980), Understanding attitudes and predicting social behavior, Englewood Cliffs, NJ, Prentice-Hall.

Allen-O’Donnell M., Cottingham M.D., Nowak T.C. et Snyder K.A. (2011), Impact of group settings and gender on meals purchased by college students, Journal of Applied Social Psychology, 41, 9, 2268-2283.

Andrews J.C., Netemeyer R.G. et Burton S. (1998), Consumer generalization of nutrient content claims in advertising, Journal of Marketing, 62, 4, 62-75.

Apfelbaum M. (1998), Risques et peurs alimentaires, Paris, Odile Jacob.

Arnould E.J. et Wallendorf M. (1994), Market-oriented ethnography: interpretation building and marketing strategy formulation, Journal of Marketing Research, 31, 4, 484-504.

Ascher F. (2005), Le mangeur hypermoderne: une figure de l'individu éclectique, Paris, Odile Jacob.

Aurier P. et Sirieix L. (2009), Marketing des produits agroalimentaires, Paris, Dunod.

Ayache M. et Dumez H. (2011a), Le codage dans la recherche qualitative: une nouvelle perspective ?, Le Libellio d'Aegis, CRG Polytechnique, 7, 2, 33-46.

Ayache M. et Dumez H. (2011b), Réflexions en forme de réponses : à propos du codage dans la recherche qualitative, Le Libellio d'Aegis, CRG Polytechnique, 7, 3, 29-34.

Bauer R. (1960), Consumer behavior as risk taking, in Dynamic marketing for a changing world, proceedings of the 43rd conference of the AMA, Chicago, American Marketing Association, 389-398.

Bech-Larsen T. et Scholderer J. (2007), Functional foods in Europe: consumer research, market experiences and regulatory aspects, Trends in Food Science \& Technology, 18, 4, 231-234.

Belk R.W. (1974), An exploratory assessment of situational effects in buyer behavior, Journal of Marketing Research (JMR), 11, 2, 156-163.

Belk R.W. (1975), Situational variables and consumer behavior, Journal of Consumer Research, 2, 3, 157-164.

Bell R. et Pliner P.L. (2003), Time to eat: the relationship between the number of people eating and meal duration in three lunch settings, Appetite, 41, 2, 215-218. 
Bergadaà M. et Urien B. (2006), Le risque alimentaire perçu comme risque vital de consommation : émergences, adaptation et gestion, Revue Française de Gestion, 32, 162, $127-144$.

Block L.G., Grier S.A., Childers T.L., Davis B., Ebert J.E.J., Kumanyika S., Laczniak R.N., Machin J.E., Motley C.M., Peracchio L., Pettigrew S., Scott M. et Van Ginkel Bieshaar M.N.G. (2011), From nutrients to nurturance: a conceptual introduction to food well-being, Journal of Public Policy \& Marketing, 30, 1, 5-13.

Block L.G. et Peracchio L.A. (2006), The calcium quandary: how consumers use nutrition labels, Journal of Public Policy \& Marketing, 25, 2, 188-196.

Boutaud J.-J. (2004), L'imaginaire de la table : convivialité, commensalité et communication, Paris, L’Harmattan.

Brée J. (2010), Marketing, alimentation et obésité infantile, Revue Management et Avenir, 37, 93-96.

Brownell K. et Warner K. (2009), The perils of ignoring history : big tobacco played dirty and millions died. How similar is big food ?, The Milbank Quarterly, 87, 1, 259-294.

Brunel O. et Pichon P.-E. (2004), Food-related risk-reduction strategies : purchasing and consumption processes, Journal of Consumer Behaviour, 3, 4, 360-374.

Bublitz M.G., Peracchio L.A., Andreasen A.R., Kees J., Kidwell B., Miller E.G., Motley C.M., Peter P.C., Rajagopal P., Scott M.L. et Vallen B. (2013), Promoting positive change : advancing the food well-being paradigm, Journal of Business Research, 66, 8, 1211-1218.

Burton S. et Creyer E.H. (2004), What consumers don't know can hurt them: consumer evaluations and disease risk perceptions of restaurant menu items, Journal of Consumer Affairs, 38, 1, 121-145.

Calandre N., Bricas N. et Sirieix L. (2009), Comment les mères perçoivent-elles les risques nutritionnels de leurs enfants? Une approche par le paradigme psychométrique au Vietnam, Economies et sociétés, 31, 1735-1760.

Camous R. (2011), Ados et prises de risques... Quelles actions de communication pour les sensibiliser aux dangers du tabac, de l'alcool, de la route, etc.?, Paris, Editions EMS Management \& Société.

Chandon P. (2012), How package design and packaged-based marketing claims lead to overeating, INSEAD Working Papers Collection, 61, 1-40.

Chandon P. et Ordabayeva N. (2009), Supersize in one dimension, downsize in three dimensions: effects of spatial dimensionality on size perceptions and preferences, Journal of Marketing Research, 46, 6, 739-753. 
Chandon P. et Wansink B. (2007a), Is obesity caused by calorie underestimation? A psychophysical model of meal size estimation, Journal of Marketing Research, 44, 1, 84-99.

Chandon P. et Wansink B. (2007b), The biasing health halos of fast-food restaurant health claims : lower calorie estimates and higher side-dish consumption intentions, Journal of Consumer Research, 34, 3, 301-314.

Chandon P. et Wansink B. (2010), Is food marketing making us fat? A multi-disciplinary review, Foundations \& Trends in Marketing, 5, 3, 1-86.

Chernev A. et Gal D. (2010), Categorization effects in value judgments : averaging bias in evaluating combinations of vices and virtues, Journal of Marketing Research, 47, 4, 738-747.

Chevallier L. (2009), Tout savoir sur les aliments : vérités et impostures, Paris, LGF Livre de Poche.

Cox D.F. (1967), Risk taking and information handling in consumer behaviour, Boston, Harvard University Press.

Cunningham S.M. (1967), The major dimensions of perceived risk, in D.F. Cox (coord.), Risk taking and information handling in consumer behaviour, Boston, Harvard University Press, 82-108.

De Castro J.M. (2010), The control of food intake of free-living humans : putting the pieces back together, Physiology \& Behavior, 100, 5, 446-453.

Douglas M. et Wildavsky A.B. (1983), Risk and culture: an essay on the selection of technical and environmental dangers, Berkeley, CA, University of California Press.

Evrard Y., Pras B., Roux E., Desmet P. et Collectif (2009), Market : fondements et méthodes des recherches en marketing, Paris, Dunod.

Faulkner G.P., Pourshahidi L.K., Wallace J.M.W., Kerr M.A., McCaffrey T.A. et Livingstone M.B.E. (2013), Perceived "healthiness " of foods can influence consumers' estimations of energy density and appropriate portion size, International journal of obesity (2005).

Ferreira C. (2006), The influence of food portion size and energy density on energy intake: implications for weight management, Journal of Risk Research, 9, 8, 851-868.

Ferrières M. (2006), Histoire des peurs alimentaires : du Moyen Age à l'aube du XXe siècle, Paris, Seuil.

Fischler C. (1990), L'homnivore, Paris, Odile Jacob.

Fischler C. (2001), La peur est dans l'assiette, Revue Française du Marketing, 183/184, 3/4, 7-10. 
Fischler C. (2011), Commensality, society and culture, Social Science Information, 50, 3-4, 528-548.

Fischler C. et Masson E. (2008), Manger: Français, Européens et Américains face à l'alimentation, Paris, Odile Jacob.

Fischler C., Merdji M., Michels S., Riou N. et Allart M.-A. (2010), Etude EuroAllégations, Paris, Protéines et BrainValue.

Fishbein M. et Ajzen I. (1975), Belief, attitude, intention and behavior : an introduction to theory and research, Boston, Addison-Wesley Publishing.

Flandrin J.L. et Montanari M. (1996), Histoire de l'alimentation, Paris, Fayard.

Frazer J.-G. (1911), Le rameau d'or, Paris, Robert Laffont (1998).

Gallen C. (2001), Le besoin de réassurance en consommation alimentaire, Revue Française du Marketing, 183/184, 3/4, 67-85.

Garg N., Wansink B. et Inman J.J. (2007), The influence of incidental affect on consumers' food intake, Journal of Marketing, 71, 1, 194-206.

Garretson J.A. et Burton S. (2000), Effects of nutrition facts panel values, nutrition claims, and health claims on consumer attitudes, perceptions of disease-related risks, and trust, Journal of Public Policy \& Marketing, 19, 2, 213-227.

Gavard-Perret M.-L., Gotteland D., Haon C. et Jolibert A. (2008), Méthodologie de la Recherche, réussir son mémoire ou sa thèse en sciences gestion, Paris, Pearson.

Gomez P. (2008), La nutrition dans les stratégies d'innovation alimentaire: de la protection du risque de santé publique à la construction de l'avantage concurrentiel, Décisions Marketing, 49, 71-83.

Gomez P. (2009a), Les heuristiques de choix nutritionnels : une étude qualitative exploratoire, in Journées de Recherche en Marketing de Bourgogne, 14, Dijon, 1-25.

Gomez P. (2009b), L'orientation régulatrice de santé : déterminants, mesure et conséquences sur les comportements de santé et le traitement de l'information, Thèse de doctorat en sciences de gestion, IAE Université de Nantes.

Grunert K.G. et Wills J.M. (2007), A review of European research on consumer response to nutrition information on food labels, Journal of Public Health, 15, 5, 385-399.

Gurviez P. (2001), Le rôle de la confiance dans la perception des risques alimentaires par les consommateurs, Revue Française du Marketing, 183/184, 3/4, 87-98.

Gurviez P. (2010), «Et là je dis attention arnaque! » Evitement et résistance des consommateurs envers une marque qui leur veut du bien, in Journées de Recherche en Marketing de Bourgogne, 11, Dijon, 56-78. 
Herman C.P., Roth D.A. et Polivy J. (2003), Effects of the presence of others on food intake : a normative interpretation, Psychological Bulletin, 129, 6, 873-886.

IREMAS (2010), Livre blanc cacophonie alimentaire et nutritionnelle, Jouy-en-Josas, Institut pour la Recherche en Marketing de l’Alimentation Santé.

Iyengar S.S. et Lepper M.R. (2000), When choice is demotivating: can one desire too much of a good thing?, Journal of Personality \& Social Psychology, 79, 6, 995-1006.

Kahneman D. et Tversky A. (1979), Prospect theory: an analysis of decision under risk, Econometrica, 47, 2, 263-291.

Kapferer J.-N. (1998), Les marques, base de la confiance?, in M. Apfelbaum (coord.), Risques et peurs alimentaires, Paris, Odile Jacob, 203-210.

Kaplan L.B., Szybillo G.J. et Jacoby J. (1974), Components of perceived risk in product purchase : a cross-validation, Journal of Applied Psychology, 59, 3, 287-291.

Keller S.B., Landry M., Olson J., Velliquette A.M., Burton S. et Andrews J.C. (1997), The effects of nutrition package claims, nutrition facts panels, and motivation to process nutrition information on consumer product evaluations, Journal of Public Policy \& Marketing, 16, 2, 256-269.

Kermisch C. (2011), Le concept du risque : de l'épistémologie à l'éthique, Paris, Tec \& Doc.

Kozup J.C., Creyer E.H. et Burton S. (2003), Making healthful food choices : the influence of health claims and nutrition information on consumers' evaluations of packaged food products and restaurant menu items., Journal of Marketing, 67, 2, 19-34.

Kytle B. et Ruggie J.G. (2005), Corporate social responsibility as risk management : z model for multinationals, Papier de recherche Corporate social responsibility as risk management, Boston, John F. Kennedy School of Government, Harvard University.

Lahlou S. (1995), Penser-manger: les représentations sociales de l'alimentation, Thèse de doctorat en psychologie sociale, Ecole des Hautes Etudes en Sciences Sociales, Paris.

Larceneux F. (2003a), Segmentation des signes de qualité : labels experientiels et labels techniques, Décisions Marketing, 29, 35-46.

Larceneux F. (2003b), Stratégies de signalisation de la qualité : l'impact des labels sur le processus de décision des consommateurs, Thèse de doctorat en sciences de gestion, Université Paris-Dauphine.

Larceneux F., Rieunier S. et Fady A. (2007), Effet de l'hyperchoix sur le consommateur et effet modérateur de la marque: une application au cas de l'horlogerie-bijouterie, Recherche et Applications en Marketing, 22, 4, 43-57.

Lardellier P. (2011), Opéra bouffe: une anthropologie gourmande de nos modes alimentaires, Paris, Editions EMS Management \& Société. 
Latham M.C. (2001), Human nutrition in the developing world, in Food and Nutrition Series, 29, Rome, FAO.

Lipovetsky G. (2006), Le bonheur paradoxal: essai sur la société d'hyperconsommation, Paris, Gallimard.

Lone T.A., Pence D., Levi A.E., Chan K.K. et Bianco-Simeral S. (2009), Marketing healthy food to the least interested consumers, Journal of Foodservice, 20, 2, 90-99.

Maddux J.E. et Rogers R.W. (1983), Protection motivation and self-efficacy : a revised theory of fear appeals and attitude change, Journal of Experimental Social Psychology, 19, 5, 469-479.

Mason C.F. (2009), Certification of socially responsible behavior: eco-labels and fair-trade coffee, Journal of Agricultural \& Food Industrial Organization, 7, 1-19.

Mathé T., Pilorin T. et Hébel P. (2008), Du discours nutritionnel aux représentations de l’alimentation, Cahier de recherche n²52, Paris, Crédoc.

McCann M.T., Wallace J.M.W., Robson P.J., Rennie K.L., McCaffrey T.A., Welch R.W. et Livingstone M.B.E. (2013), Influence of nutrition labelling on food portion size consumption, Appetite, 65, 153-158.

McFerran B., Dahl D.W., Fitzsimons G.J. et Morales A.C. (2013), I'll have what she's having: effects of social influence and body type on the food choices of others, Journal of Consumer Research, 36, 6, 915-929.

Merdji M. (2006), L’imaginaire du dégoût: une approche anthropologique de l'univers émotionnel de l'alimentation, in M. Kalika et P. Romelaer (coord.), Recherches en Management et Organisation, Paris, Economica, 179-194.

Merdji M. (2010), Consumers' perception and uses of nutritional and health claims, in Nutreating Conference: Food, Consumer and Health Claims, AgroParisTech.

Merdji M. (2012), Profils nutritionnels, in J.-P. Poulain (coord.), Dictionnaire des cultures alimentaires, Presses Universitaires de France.

Méric J., Pesqueux Y. et Solé A. (2009), La société du risque: analyse et critique, Paris, Economica.

Michel G. (2004), Au coeur de la marque: les clés du management de la marque, Paris, Dunod.

Mishra A., Mishra H. et Masters T.M. (2012), The influence of bite size on quantity of food consumed: a field study, Journal of Consumer Research, 38, 5, 791-795.

Mitchell V.-W. (1999), Consumer perceived risk : conceptualisations and models, European Journal of Marketing, 33, 1/2, 6-195.

Moon W. et Balasubramanian S. (2003), Willingness to pay for non-biotech foods in the U.S. and U.K., Journal of Consumer Affairs, 37, 2, 317-339. 
Moore E.S. et Rideout V.J. (2007), The online marketing of food to children : is it just fun and games?, Journal of Public Policy \& Marketing, 26, 2, 202-220.

Mori D., Chaiken S. et Pliner P. (1987), «Eating lightly » and the self-presentation of femininity, Journal of Personality and Social Psychology, 53, 4, 693-702.

Nestle M. (2007), Food politics: how the food industry influences nutrition and health, Berkeley, CA, University of California Press.

OMS (1946), Préambule à la constitution de l’Organisation Mondiale de la Santé, in Actes officiels de l'Organisation Mondiale de la Santé, 2, 100.

OMS (1990), WHO global programme on AIDS : social and behavioural research, Disasters, 14, 3, 276-281.

OMS et FAO (2003), Régime alimentaire, nutrition et prévention des maladies chroniques, in Série de rapports techniques, 916, Genève, OMS.

Parker J.R. et Schrift R.Y. (2011), Rejectable choice sets: how seemingly irrelevant nochoice options affect consumer decision processes, Journal of Marketing Research, $48,5,840-854$.

Pennings J.M.E., Wansink B. et Meulenberg M.T.G. (2002), A note on modeling consumer reactions to a crisis: the case of the mad cow disease, International Journal of Research in Marketing, 19, 1, 91-100.

Pidgeon N., Kasperson R.E. et Slovic P. (2003), The social amplification of risk, Cambridge, UK, Cambridge University Press.

Pliner P., Bell R., Hirsch E.S. et Kinchla M. (2006), Meal duration mediates the effect of « social facilitation » on eating in humans, Appetite, 46, 2, 189-198.

Pollan M. (2009), In defense of food : an eater's manifesto, New York, Penguin Books.

Poulain J.-P. (2002), Sociologies de l'alimentation: les mangeurs et l'espace social alimentaire, Paris, Presses Universitaires de France.

Rogers R.W. (1975), A protection motivation theory of fear appeals and attitude change, The Journal of Psychology, 91, 1, 93-114.

Rosa E.A. (2003), The logical structure of the social amplification of risk framework (SARF) : metatheoretical foundations and policy implications, in N. Pidgeon, R.E. Kasperson et P. Slovic (coord.), The social amplification of risk, Cambridge, UK, Cambridge University Press, 47-79.

Roselius R. (1971), Consumer rankings of risk reduction methods, Journal of Marketing, 35, 1, 56-61.

Rozin P. (2005a), The meaning of « natural », Psychological Science, 16, 8, 652-658. 
Rozin P. (2005b), The meaning of food in our lives: a cross-cultural perspective on eating and well-being, Journal of Nutrition Education \& Behavior, 37, 107-112.

Rozin P. (2006), Naturalness judgments by lay Americans: process dominates content in judgments of food or water acceptability and naturalness, Judgment and Decision Making, 1, 2, 91-97.

Rozin P., Fischler C., Shields C. et Masson E. (2006), Attitudes towards large numbers of choices in the food domain : a cross-cultural study of five countries in Europe and the USA, Appetite, 46, 3, 304-308.

Rozin P., Millman L. et Nemeroff C. (1986), Operation of the laws of sympathetic magic in disgust and other domains, Journal of Personality and Social Psychology, 50, 4, 703-712.

Rozin P., Spranca M., Krieger Z., Neuhaus R., Surillo D., Swerdlin A. et Wood K. (2004), Preference for natural : instrumental and ideational/moral motivations, and the contrast between foods and medicines, Appetite, 43, 2, 147-154.

Saulais L., Doyon M., Ruffieux B. et Kaiser H. (2012), Consumer knowledge about dietary fats : another French paradox?, British Food Journal, 114, 1, 108-120.

Scheibehenne B., Greifeneder R. et Todd P.M. (2010), Can there ever be too many options? A meta-analytic review of choice overload, Journal of Consumer Research, 37, 3, 409-425.

Schuldt J.P., Muller D. et Schwarz N. (2012), The « fair trade » effect health halos from social ethics claims, Social Psychological and Personality Science, 3, 5, 581-589.

Schwartz B. (2004), The paradox of choice : why more is less, New York, Harper Perennial.

Sharpe K.M., Staelin R. et Huber J. (2008), Using extremeness aversion to fight obesity: policy implications of context dependent demand, Journal of Consumer Research, 35, 3, 406-422.

Siegrist M. (2003), Perception of gene technology, and food risks : results of a survey in Switzerland, Journal of Risk Research, 6, 1, 45-60.

Simon H.W., Berkowitz N.N. et Moyer R.J. (1970), Similarity, credibility, and attitude change : a review and a theory, Psychological Bulletin;Psychological Bulletin, 73, 1, 1-16.

Singh J. et Sirdeshmukh D. (2000), Agency and trust mechanisms in consumer satisfaction and loyalty judgments, Journal of the Academy of Marketing Science, 28, 1, 150-167.

Sirieix L. (1999), La consommation alimentaire: problématiques, approches et voies de recherche, Recherche et Applications en Marketing, 14, 3, 41-58.

Sirieix L. et Dubois P.-L. (1999), Vers un modèle qualité-satisfaction intégrant la confiance, Recherche et Applications en Marketing, 14, 3, 1-22. 
Slovic P. (1987), Perception of risk, Science, 236, 4799, 280 -285.

Slovic P. et Peters E. (2006), Risk perception and affect, Current Directions in Psychological Science, 15, 6, 322-325.

Stroebele N. et De Castro J.M. (2004), Effect of ambience on food intake and food choice, Nutrition, 20, 9, 821-838.

Taylor J.W. (1974), The role of risk in consumer behavior, Journal of Marketing, 38, 2, 54-60.

Tversky A. et Kahneman D. (1992), Advances in prospect theory : cumulative representation of uncertainty, Journal of Risk \& Uncertainty, 5, 4, 297-323.

Verbeke W., Vanhonacker F., Frewer L.J., Sioen I., De Henauw S. et Van Camp J. (2008), Communicating risks and benefits from fish consumption: impact on Belgian consumers' perception and intention to eat fish, Risk Analysis: An International Journal, 28, 4, 951-967.

Volle P. (1995), Le concept de risque perçu en psychologie du consommateur : antécédents et statut théorique, Recherche et Applications en Marketing, 10, 1, 39-56.

Wansink B. (2006), Mindless eating: why we eat more than we think, New York, Bantam Dell.

Wansink B. et Chandon P. (2006), Can « low-fat » nutrition labels lead to obesity?, Journal of Marketing Research, 43, 605-617.

Werle C., Boesen-Mariani S., Gavard-Perret M.-L. et Berthaud S. (2012), Prévention de l'obésité auprès des adolescents: l'efficacité de l'argument «risque social » sur les intentions et comportements alimentaires, Recherche et Applications en Marketing, 27, 3, 3-29.

Wilcox K., Vallen B., Block L. et Fitzsimons G.J. (2009), Vicarious goal fulfillment: when the mere presence of a healthy option leads to an ironically indulgent decision, Journal of Consumer Research, 36, 3, 380-393.

Zingg A., Cousin M.-E., Connor M. et Siegrist M. (2013), Public risk perception in the total meat supply chain, Journal of Risk Research, 16, 8, 1005-1020. 